\title{
Novel $\gamma$-sarcoglycan interactors in murine muscle membranes
}

\author{
Tara C. Smith', Georgios Vasilakos², Scott A. Shaffer ${ }^{3,4}$, Jason M. Puglise², Chih-Hsuan Chou², \\ Elisabeth R. Barton ${ }^{2^{*}}$ and Elizabeth J. Luna ${ }^{1 *}$ (D)
}

\begin{abstract}
Background: The sarcoglycan complex (SC) is part of a network that links the striated muscle cytoskeleton to the basal lamina across the sarcolemma. The SC coordinates changes in phosphorylation and $\mathrm{Ca}^{++}$-flux during mechanical deformation, and these processes are disrupted with loss-of-function mutations in gamma-sarcoglycan (Sgcg) that cause Limb girdle muscular dystrophy 2C/R5.

Methods: To gain insight into how the SC mediates mechano-signaling in muscle, we utilized LC-MS/MS proteomics of SC-associated proteins in immunoprecipitates from enriched sarcolemmal fractions. Criteria for inclusion were coimmunoprecipitation with anti-Sgcg from C57BL/6 control muscle and under-representation in parallel experiments with Sgcg-null muscle and with non-specific lgG. Validation of interaction was performed in co-expression experiments in human RH30 rhabdomyosarcoma cells.

Results: We identified 19 candidates as direct or indirect interactors for Sgcg, including the other 3 SC proteins. Novel potential interactors included protein-phosphatase-1-catalytic-subunit-beta (Ppp1cb, PP1b) and $\mathrm{Na}^{+}-\mathrm{K}^{+}-\mathrm{Cl}^{-}$-co-transporter NKCC1 (SLC12A2). NKCC1 co-localized with Sgcg after co-expression in human RH30 rhabdomyosarcoma cells, and its cytosolic domains depleted Sgcg from cell lysates upon immunoprecipitation and co-localized with Sgcg after detergent permeabilization. NKCC1 localized in proximity to the dystrophin complex at costameres in vivo. Bumetanide inhibition of NKCC1 cotransporter activity in isolated muscles reduced SC-dependent, strain-induced increases in phosphorylation of extracellular signal-regulated kinases 1 and 2 (ERK1/2). In silico analysis suggests that candidate SC interactors may cross-talk with survival signaling pathways, including p53, estrogen receptor, and TRIM25.
\end{abstract}

Conclusions: Results support that NKCC1 is a new SC-associated signaling protein. Moreover, the identities of other candidate SC interactors suggest ways by which the SC and NKCC1, along with other Sgcg interactors such as the membrane-cytoskeleton linker archvillin, may regulate kinase- and $\mathrm{Ca}^{++}$-mediated survival signaling in skeletal muscle.

Keywords: Limb girdle muscular dystrophy, Skeletal muscle, Sarcolemma, Archvillin, Svil, PP1 $\beta / \delta$, Sarcoglycans, NKCC1

*Correspondence: erbarton@ufl.edu; Elizabeth.Luna@umassmed.edu 1 Department of Radiology, Division of Cell Biology \& Imaging, University of Massachusetts Medical School, Worcester, MA, USA

${ }^{2}$ Applied Physiology \& Kinesiology, College of Health \& Human Performance, University of Florida, Gainesville, FL, USA

Full list of author information is available at the end of the article

\section{Background}

The dystrophin glycoprotein complex (DGC) is a major sarcolemmal complex that tethers the extracellular matrix $(\mathrm{ECM})$ to the intracellular actin cytoskeleton $[1$, 2]. Together with the integrin-associated focal adhesion complex [3, 4], the DGC stabilizes the membrane, senses mechanical stress, and transmits "outside-in" information

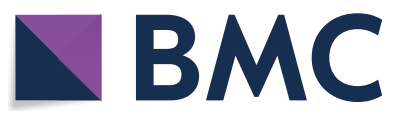

(c) The Author(s) 2022. Open Access This article is licensed under a Creative Commons Attribution 4.0 International License, which permits use, sharing, adaptation, distribution and reproduction in any medium or format, as long as you give appropriate credit to the original author(s) and the source, provide a link to the Creative Commons licence, and indicate if changes were made. The images or other third party material in this article are included in the article's Creative Commons licence, unless indicated otherwise in a credit line to the material. If material is not included in the article's Creative Commons licence and your intended use is not permitted by statutory regulation or exceeds the permitted use, you will need to obtain permission directly from the copyright holder. To view a copy of this licence, visit http://creativecommons.org/licenses/by/4.0/. The Creative Commons Public Domain Dedication waiver (http://creativeco mmons.org/publicdomain/zero/1.0/) applies to the data made available in this article, unless otherwise stated in a credit line to the data. 
to the nucleus via signaling proteins, including phosphorylated signaling kinases $[5,6]$. The dominant DGC connection to actin is via dystrophin, which links the actin cytoskeleton to dystroglycan, and in turn binds laminin in the ECM $[1,2]$. The sarcoglycan complex (SC) is a sub-complex of the DGC, comprised of $\alpha-, \beta-, \delta$-, and $\gamma$-sarcoglycans (Sgca, Sgcb, Sgcd, Sgcg) in skeletal muscle [7]. These proteins form a heterotetramer, each having a short intracellular domain, a single membrane-spanning $\alpha$-helix, and a large extracellular domain. Sarcolemmal DGC proteins are concentrated at costameres, which are associated with the mouths of T-tubules and contain a multitude of signaling proteins [2, 8-13].

Deficiencies in many DGC proteins are causal for muscular dystrophies in humans and characterized by disarranged costameres, progressive muscle loss, and shortened lifespan [14, 15]. Loss of any SC protein in humans or mice leads to the significant reduction of the entire sub-complex and to a limb girdle muscular dystrophy (LGMD) [15]. LGMDs are characterized by severe pathology, including elevated serum creatine kinase levels, cycles of muscle degeneration and regeneration, and extensive fibrosis, yet there is variable mechanical fragility [16, 17]. Mice lacking a functional SC exhibit altered signaling through the mitogen-activated protein kinases (MAPK), including extracellular signal-regulated kinases 1 and 2 (ERK1/2) [18-21] and p38 [22], as well as increased levels of intracellular calcium ions [23] during eccentric contractions (ECC) of skeletal muscle. Although the signaling pathways have not been elucidated, Sgcg tyrosine- 6 is required for SC-dependent changes in the level of phosphorylated ERK1/2 [18, 20, 24], and mutation of Sgcg-Y6 is associated with severe auto-recessive muscular dystrophy (reviewed in [5]). Yeast two-hybrid interactors have been described for the cytosolic N-termini of the four SC proteins [25, 26], but proteomic analyses have been confounded by the relative scarcity of these proteins in muscle.

One candidate interaction partner is archvillin [27], a membrane-associated cytosolic protein that binds directly to dystrophin, interacts with Sgcg in yeast twohybrid assays, and is required for the structural integrity of human muscles [26, 28]. Archvillin is implicated in ERK1/2-regulated survival signaling in muscle [29], as is Sgcg. Smooth muscle archvillin scaffolds B-Raf and ERK1/2 to promote ERK signaling [30]. In skeletal muscle, Sgcg regulates archvillin association with P-ERK1/2 following ECC, which is ablated in the absence of Sgcg or dystrophin, and leads to heightened basal P-ERK1/2, increased nuclear P-ERK1/2 after active mechanical perturbation, and an uncoupled signaling response to passive stretch [26]. A nonmuscle isoform (supervillin, SV1), which is encoded by the same Svil gene as archvillin [27,
31], regulates focal adhesion formation and cell survival [32-34].

While it is clear that Sgcg and archvillin coordinate to modulate mechanical signaling in muscle, it leaves open the possibility for additional direct or indirect protein interactors to participate in these actions. To look for such Sgcg-associated proteins, we developed a mediumthroughput method for carrying out co-IPs of Sgcg and other sarcolemmal proteins. We identified 16 novel candidate Sgcg interactors by LC MS-MS analyses of co-IPs from single mouse muscles with and without the SG complex. We confirmed direct binding of the Sgcg N-terminus with the archvillin $\mathrm{C}$-terminus and demonstrated an interaction of $\mathrm{SC}$ with $\mathrm{N}$ - and $\mathrm{C}$-terminal cytoplasmic domains of the $\mathrm{Na}^{+}-\mathrm{K}^{+}-2 \mathrm{Cl}^{-}$-cotransporter (NKCC1). We also report for the first time that NKCC1 immunofluorescence overlaps with that of costameric dystrophin at the sarcolemma and that NKCC1 cotransporter activity is required for the ECC-induced increases in activated ERK seen in $\mathrm{Sgcg}^{-/-}$mouse muscle. These results are the first to localize NKCC1 at or near muscle costameres and to implicate this channel in SG-mediated signaling.

\section{Methods \\ Affinity-purified rabbit polyclonal anti- $\gamma$-sarcoglycan and anti-archvillin}

Rabbit polyclonal antibodies specific for extracellular residues 72-290 in murine $\gamma$-sarcoglycan (Sgcg, NCBI reference sequence NP_036022.1) and for murine archvillin (mAV, Swiss Protein Q8K463.1) amino acids 121-568 were generated against bacterially expressed proteins at Cocalico Biologicals, Inc. (Stevens, PA). Affinity purification employed columns containing the corresponding GST-tagged proteins and protocols detailed previously for high-avidity antibodies against human supervillin [35]. After a series of stringent washes, high-avidity antibodies were eluted from a column with covalently bound immunogen using $4.5 \mathrm{M} \mathrm{MgCl}_{2}, 72.5 \mathrm{mM}$ Tris$\mathrm{HCl} \mathrm{pH} \mathrm{6.0-7.0.} \mathrm{PCR} \mathrm{templates} \mathrm{were} \mathrm{a} \mathrm{murine} \mathrm{Sgcg}$ plasmid from Dr. Elizabeth McNally [36] and an EGFPtagged mAV plasmid [27], respectively. PCR primers included the underlined sites for directed restriction cloning, as shown in Additional file 1, Supplementary Table S1. PCR products were generated with Pfu Turbo DNA polymerase (Agilent Technologies, Santa Clara, CA) according to the manufacturer's directions, gel purified, cloned into TOPO-pCR2.1 vector (ThermoFisher Scientific, Waltham, MA), and verified by DNA sequencing. Coding sequences for Sgcg and $\mathrm{mAV}$ were recovered from doubly digested vectors and ligated into identically digested pGEX-6P-1 (Sigma-Aldrich, St. Louis, MO). Soluble GST-mAV was isolated from Rosetta 2(DE3)pLysS chemically competent bacteria (EMD-Millipore-Sigma, 
Burlington, MA) induced overnight with $0.2 \mathrm{mM}$ isopropyl b-D-thiogalactopyranoside and purified on glutathione-Sepharose $^{\mathrm{TM}}$ (Sigma-Aldrich). The $\sim 26-\mathrm{kDa}$ $\mathrm{mAV}$ immunogen was generated by cleavage with PreScission Protease (Sigma-Aldrich), dialyzed to remove residual glutathione, and purified by removing GST with a second glutathione-Sepharose column, followed by electrophoresis on a $15 \%$ acrylamide SDS-gel [37]. Because GST-Sgcg was incorporated into inclusion bodies and was poorly cleaved after urea renaturation, Histagged Sgcg was generated by cloning the BamHI and EcoRI DNA fragment into doubly cut pET-30a vector (Sigma-Aldrich). Urea-solubilized His-Sgcg was purified on Ni-NTA agarose columns (Qiagen, Germantown, $\mathrm{MD})$, as described [38], eluting with 50-100 mM histidine in a step gradient of $10-250 \mathrm{mM}$ histidine in column buffer (7 M urea, $5 \mathrm{mM}$ glycine, $50 \mathrm{mM}$ Tris- $\mathrm{HCl}$, $\mathrm{pH} 8.0$, $0.3 \mathrm{M} \mathrm{NaCl}, 0.5 \mathrm{mM}$ DTT, $1 \mathrm{mM}$ PMSF). The $\sim 29-\mathrm{kDa}$ His-Sgcg remained soluble after dialysis against $1 \mathrm{mM}$ DTT, PBS, pH 7.4, and was used as immunogen without further purification. Rabbits were boosted until high titers were observed on immunoblots, and each antibody was affinity purified with the corresponding GST-tagged protein covalently bound to $\mathrm{CNBr}$-activated Sepharose (Sigma-Aldrich, C9142) [35]. After affinity purification, the rabbit anti-mAV antibody was used at 1:5000$1: 10000$ for immunoblots (IB), and the rabbit anti-Sgcg was used at 1:5000 for IB and at 1:200 for immunofluorescence microscopy (IF).

\section{Commercial antibodies}

Dilutions refer to those used for IB, IF, and immunoprecipitations (IP). Murine monoclonal anti-HA-Tag (6E2, \#2367; IB: 1:1500, IF: 1:200) and anti-DYKDDDDK/Flag epitope (9A3, \#8146; IB: 1:1000, IF: 1:200) antibodies were obtained from Cell Signaling Technologies (Beverly, MA). Murine monoclonal hybridoma supernatants were obtained from the Developmental Studies Hybridoma Bank (Iowa City, IA) to stain dystrophin (MANDRA1, 7A10, IB: 1:1000; MANDYS1, 3B7, IF 1:10) [39], $\alpha$-sarcoglycan (IVD3(1)A9; IB 1:50), $\beta$-dystroglycan (MANDAG1 (7A11); IB 1:200), contractile myosins II (A4.1025; IB 1:100) [40, 41], troponin T (JLT12; IB 1:300) and SERCA1 (CaF2-5D2; IB 1:70). Rabbit monoclonal anti-HA (C29F4, \#3724; IB: 1:1000, IP: $18 \mu \mathrm{l} / 60 \mu \mathrm{l}$ of Dynabeads) and affinity-purified rabbit polyclonal antiDYKDDDDK (\#2368; IB: 1:1000, IP: $18 \mu \mathrm{l}$ per $60 \mu \mathrm{l}$ of Dynabeads) antibodies were from Cell Signaling Technologies. Rabbit monoclonal anti-PP1 $\beta$ was from Abcam (\#ab53315, Cambridge, MA, USA; IB: 1:1000, IF: 1:100). Affinity-purified rabbit polyclonal antibodies against MYPT2 (\#13366-1-AP; IB: 1:1000), NKCC1 (\#138841-AP; IB: 1:1000, IF: 1:500) and Sgcg (\#18102-1-AP; IB:
1:1000, IF: 1:100) were purchased from Proteintech Group Inc. (Rosemont, IL). Other rabbit polyclonal antibodies were anti-PP1 $\beta$ (\#LS-C482256, Life Span BioSciences Inc., Seattle, WA; IB: 1:500), anti-NKCC1 (\#ANT-071, Alomone Labs, Radassah Einkerem, Jerusalem, Israel; IB: 1:500, IF: 1:50), and anti-ERK1/2 (\#ABS44, EMD Millipore Sigma; IB: 1:1000). Isotype-specific control antibodies were rabbit IgG (EMD-Millipore \#12370) and mouse IgG2a (\#401502, BioLegend, San Diego, CA). For chemiluminescence detection in IB, we used horseradish peroxidase-conjugated donkey anti-mouse (\#715-035-150) and donkey anti-rabbit (\#711-035-152) secondary antibodies from Jackson ImmunoResearch (West Grove, PA) at 1:20,000 dilution. IB with nearinfrared fluorescent detection used primary antibodies from Cell Signaling Technology against phospho-ERK1/2 (\#9101; 1:2000) and total-ERK1/2 (\#9102; 1:2000). Primary antibodies against NKCC1 were rabbit T-NKCC (1:2000, \#13884-1-AP; Proteintech) and sheep polyclonal antibodies against P-NKCC1: phospho Thr 203, Thr 207, Thr 212 (IB: 1:1000, \#S763B) and phospho Thr 212, Thr 217 (IB: 1:1000, \#S603D), both from the MRC Protein Phosphorylation and Ubiquitylation Unit, University of Dundee, (United Kingdom). Secondary antibodies (\#925-68071, anti-rabbit and \#925-32214, anti-mouse, both 1:15,000) were from Li-Cor Biosciences (Lincoln, NE). IF signals from transfected RH30 cells were visualized with goat-anti-mouse Alexafluor 350 (\#A 21049) and goat anti-rabbit Alexafluor 568 (\#A11036) conjugated antibodies, and the actin cytoskeleton was stained with Alexafluor 350- or 488-conjugated phalloidin (all from ThermoFisher Scientific, diluted 1:500). Nuclei were stained using 4,6-diamidine-2-phenylindole dihydrochloride (DAPI, Sigma-Aldrich).

\section{Animal models}

All animals used in this study were bred, housed, and treated in accordance with standards set by the Animal Care and Use Committees at the University of Florida and the University of Massachusetts Medical School. Mice with a null mutation of the $S g c g$ gene were generated, bred, and phenotyped, as described previously [16]. Mice lacking the long muscle-specific archvillin (SV2) splice-form encoded by the Svil gene were generated by Ingenious Targeting Laboratory (Ronkonkoma, NY). The biochemical and functional consequences of this mutation are under investigation and will be described in detail elsewhere. In brief, the guide RNAs $5^{\prime}$-TGTAGG GCGATCCAAAGAAGAAG- $3^{\prime}$ and $5^{\prime}$-TCTTCAATG CTTACCTGGCTCGG- $3^{\prime}$ and CRISPR/Cas 9 were used to generate a targeting vector in which the 609-nt exon 3 in the Svil gene, called exon 2 in the initial cDNA cloning paper [27], was replaced by an ivNeo selection cassette 


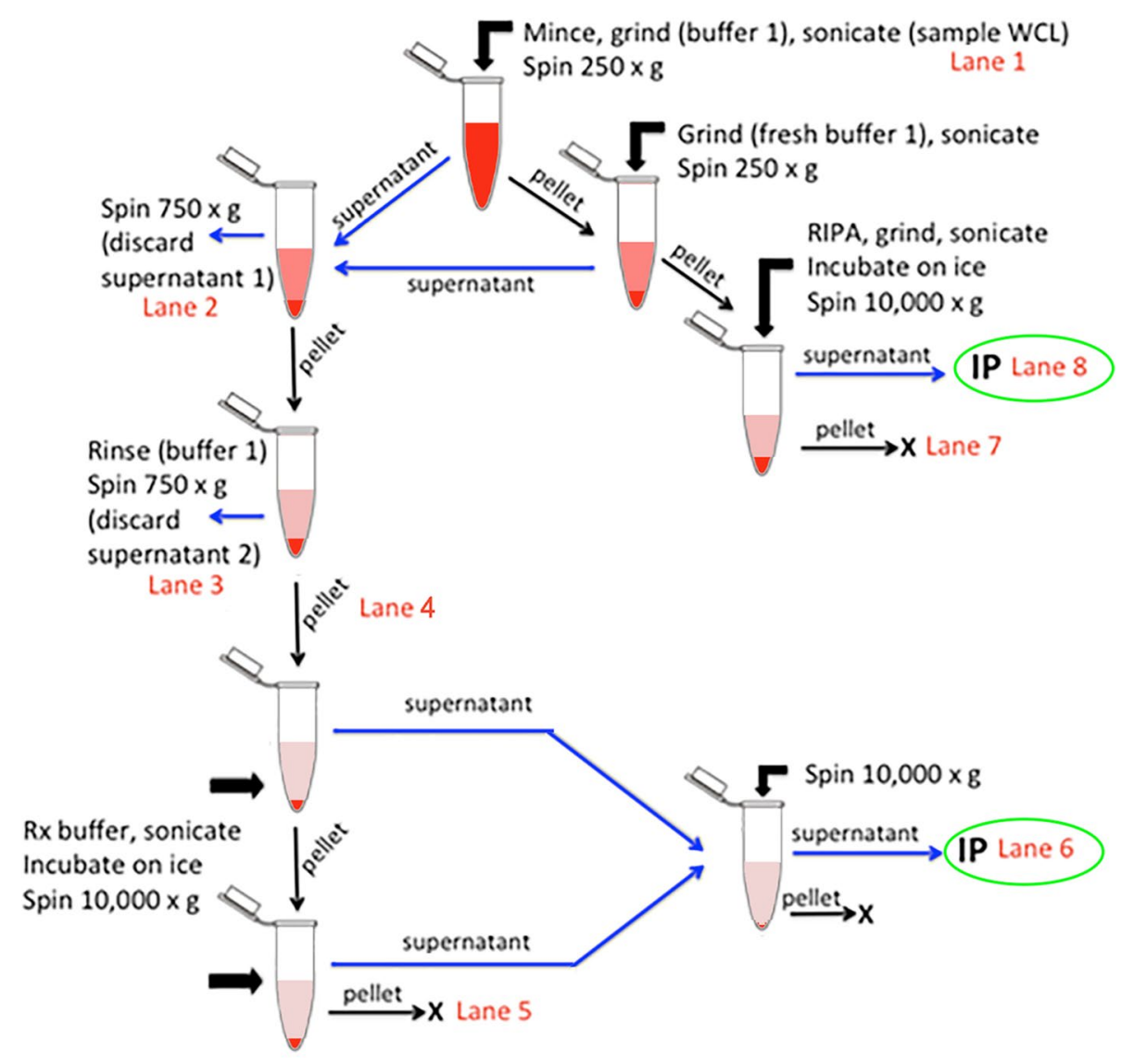

Fig. 1 Flow chart for rapid membrane enrichment from mouse muscle. Skeletal muscle was fractionated as shown and described in detail in "Methods." Lane numbers refer to analyses for total protein and immunoblotted sarcolemmal proteins, as shown in Fig. 2. Immunoprecipitations (IP, green ovals) with anti-Sgcg antibody were performed on extracts in either Rx buffer (high salt, 0.5\% IGEPAL CA-630 in Rx buffer; lane 6) or with RIPA buffer (1\% IGEPAL CA-630, 0.5\% deoxycholate, $0.1 \%$ SDS; lane 8). WCL, whole cell lysate

flanked by $759 \mathrm{bp}$ and 838 bp of genomic sequence [42]. The targeting construct was verified by DNA sequencing, linearized with NotI, and electroporated into C57BL/6 embryonic stem (ES) cells. Targeted C57BL/6 FLP ES cells were microinjected into Balb/c blastocysts, and the resulting chimeras with a high percentage of black coat color were mated to $\mathrm{C} 57 \mathrm{BL} / 6$ (WT) mice to generate germline Neo-deleted mice, which were identified by PCR screening of tail DNA. Neo-deleted mice were backcrossed four times with $\mathrm{C} 57 \mathrm{BL} / 6 \mathrm{NCrl}$ mice (Charles River Laboratories, Wilmington, MA) and screened by PCR for the absence of the FLP transgene and for proper integration at the Svil locus. Immunoblotting with rabbit polyclonal antibodies against archvillin $\mathrm{N}$-terminal sequences confirmed the absence of full-length archvillin in skeletal muscle (Additional file 2, Fig. S1A).

\section{Mouse muscle plasma membranes}

Mouse gastrocnemius muscles were dissected and trimmed of extraneous tissue, cut into two $\sim 100 \mathrm{mg}$ pieces (wet tissue mass), and flash frozen in liquid nitrogen. To prepare for co-IP, one piece of frozen gastrocnemius muscle was very finely minced with a razor blade at room temperature on weighing paper while thawing, transferred to a $1.5-\mathrm{ml}$ plastic mortar tube (\#7495200090, Kimble-Kontes, DWK Life Sciences, Millville, NJ), and processed according to a novel method adapted from those used to detect glucose transporter 4 [43], and other muscle membrane proteins [44-49] (Fig. 1). Minced muscles were covered with $900 \mu \mathrm{l}$ of Buffer $1(50 \mathrm{mM}$ Tris, pH 8.0, $0.5 \mathrm{mM}$ DTT, 0.1\% NP-40, 10\% glycerol, and protease inhibitor cocktail (Sigma-Aldrich \#P8340) and lysed by manual grinding for $5-10 \mathrm{~s}$ with a blue plastic Kimble-Kontes pestle. Up to 4 samples were processed at one time, and all were stored on ice between steps. When all samples in a group were ground, they were immediately sonicated for 30 pulses with a microtip probe on a Branson Sonifier Cell Disrupter (Emerson Electric, Danbury, CT) with settings of $25 \%$ duty cycle, output $~ 1.5$. A $100-\mu \mathrm{l}$ portion of each sample was taken for gel analysis 
(Muscle Lysate, WCL). The remaining volumes were centrifuged at $250 \times g$ for $1 \mathrm{~min}$ at $4{ }^{\circ} \mathrm{C}$. The cloudy supernatant was removed to a fresh $1.5-\mathrm{ml}$ polypropylene microcentrifuge tube, and $450 \mu \mathrm{l}$ of Buffer 1 was added to the remaining pellet, which was again ground manually for 5-10 s and sonicated. After another $1 \mathrm{~min}$ centrifugation at $250 \times g$, this also-cloudy supernatant was combined with the first and centrifuged together at $4{ }^{\circ} \mathrm{C}$ for 10 min at $750 \times g$. The remaining $250 \times g$ pellet was homogenized in an equivalent volume of $2 x$ RIPA1 buffer (100 mM MOPS, pH 7.5, 0.3 M NaCl, 2\% IGEPAL CA-630, $1 \%$ deoxycholic acid, $0.2 \%$ SDS, plus protease inhibitors) and incubated on ice while the other fractions were further processed; this incubation period was $\geq 25 \mathrm{~min}$ before sonication (see below). The supernatant from the $750 \times g$ spin was removed (Discard supernatant 1 ), and the remaining (white and somewhat fluffy) membrane pellet was resuspended by vortexing in $450 \mu \mathrm{l}$ of Buffer 1. The membranes were again sedimented at $750 \times g$ for $10 \mathrm{~min}$. The supernatant was removed (Discard supernatant 2), and the pelleted membranes were resuspended by briefly vortexing in $600 \mu \mathrm{l}$ of cold Rx Buffer $(25 \mathrm{mM}$ MOPS, pH 7.5, 0.1 M NaCl, $0.5 \mathrm{M} \mathrm{KCL}, 10 \mathrm{mM} \mathrm{MgCl}$, $5 \mathrm{mM}$ ATP (freshly made), $60 \mathrm{mM}$ octylglucopyranoside, $0.5 \%$ IGEPAL CA-630, 10\% glycerol, and protease inhibitors). The membranes were then sonicated three times, as above, with an incubation on ice of $1 \mathrm{~min}$ in between, until the suspension was slightly pearly. The pellets from the RIPA1-solubilized membranes also were sonicated once at this time, and both fractions were incubated on ice for $10 \mathrm{~min}$ after the last sonication. All tubes were centrifuged for $10 \mathrm{~min}$ at $10,000 \times g$, and the supernatants removed to fresh tubes. The $\mathrm{Rx}$ membrane pellet was resuspended by briefly vortexing in $400 \mu \mathrm{l}$ of $\mathrm{Rx}$ buffer and sonicated once before centrifugation at $10,000 \times g$ for $10 \mathrm{~min}$. This final Rx pellet was resuspended in an equal volume of 2x Laemmli Sample Buffer [37]. The two Rx supernatants were combined and subjected to a final centrifugation at $10,000 \times g$ for $10 \mathrm{~min}$. The RIPA1 pellet was resuspended in a Laemmli sample buffer volume equivalent to that of the corresponding RIPA supernatant.

\section{Light and confocal microscopy}

Phase images of extracted muscle membranes were obtained with a Leica DMI 6000B inverted fluorescence microscope with a Leica DFC 365 FX camera, a Leica HCX PL Fluotar 10x/0.30 PH1 lens, and Leica Application Suite 3.2.0.9625 software (Leica Microsystems, Exton, PA). Immunofluorescent images were taken on the same system using a Leica HC PL APO 63x/1.40-0.60 oil lens. Images of fluorescently stained muscle sections were obtained on a Leica SP5 (II) AOBS laser scanning confocal microscope with a HCX PL APO CS 40.0 ×/1.30 oil UV lens and using Leica Application Suite Advanced Fluorescence (LAS-AF) 2.7.3.9723 software (Leica Microsystems CMS GmbH, Mannheim, Germany). Optical $z$-sections of $0.29 \mu \mathrm{m}$ were obtained sequentially for each color channel through the muscle sample. Selected sequential sections comprising no more than $1.2 \mu \mathrm{m}$ of $z$-thickness were processed using the Maximum Intensity Projection function in the software. All images were exported as TIF files, then uniformly adjusted and assembled with Adobe Photoshop CS3 software (Adobe Systems, Inc., San Jose, CA).

\section{Immunoblotting and analyses}

Proteins were electrotransferred overnight onto Amersham $^{\mathrm{TM}} \operatorname{Protran}^{\mathrm{TM}} 0.45-\mu \mathrm{m}$ nitrocellulose membranes (GE Healthcare Life Science, Marlborough, MA; \#10600002). Chemiluminescent signals were visualized by SuperSignal West Pico or Femto reagents (ThermoFisher Scientific) on a Chemidoc MP Imaging System, using ImageLab ${ }^{\mathrm{TM}}$ software, version 4.1 (Bio-Rad Life Science Research, Hercules, CA). Densitometry of protein bands was determined using GelQuant.Net (version 1.7.8, BiochemLabSolutions.com; University of California, San Francisco, CA) software. Ratios of Unbound to Input signals were calculated using Microsoft Excel (Microsoft, Redmond, WA) and analyzed using GraphPad Prism 8.4.0 software (GraphPad Software, L.L.C., La Jolla, CA). Comparisons of multiple datasets were carried out using standard or non-parametric one-way ANOVAs, as indicated in the figure legends. Immunoblots were uniformly adjusted and assembled with Adobe Photoshop CS6 software.

For $\mathrm{P} / \mathrm{T}$-ERK1/2 and P/T-NKCC1 immunoblotting, extensor digitorum longus (EDL) muscles and tibialis anterior (TA) muscles were powdered using a mortar and pestle in dry ice and homogenized in RIPA2 buffer supplemented with PMSF and inhibitors of proteases (\#P8340; Sigma-Aldrich) and phosphatases (\#P5726; Sigma-Aldrich). Homogenates were incubated on ice for $60 \mathrm{~min}$ and centrifuged at $15,000 \times \mathrm{g}$ for $15 \mathrm{~min}$. Protein quantifications of muscle lysates were determined by the Bradford assay (\#1863028; Thermo Fisher Scientific). For P/T-ERK1/2 analyses, EDL lysates (20 $\mu$ g protein) were loaded in 4-12\% Bis-Tris Midi Protein Gels (\#WG1402A, Thermo Fisher Scientific) and electrotransferred to nitrocellulose. Each membrane was blocked for $90 \mathrm{~min}$ at room temperature with 5\% BSA (BP9703100, Thermo Fisher Scientific) in TBS. Blots were incubated with antiphospho-ERK1/2 and anti-total-ERK1/2 primary antibodies overnight at $4{ }^{\circ} \mathrm{C}$, washed and incubated for 90 min at room temperature with secondary antibodies ( $\mathrm{Li}$ Cor Biosciences).

For P/T-NKCC1 analysis, TA lysates (20 $\mu$ g protein) were loaded in 3-8\% Bis-Tris Gel 1.0 mm (WG1002BOX; 
Thermo Fisher Scientific) and transferred to ImmobilonFL PVDF, $0.45-\mu \mathrm{m}$ porosity (IPFL00010, EMD-Millipore) for $16 \mathrm{~h}$ at $40 \mathrm{~mA}$ in $10 \%$ methanol, $0.01 \%$ SDS, $0.1 \%$ NuPage Antioxidant (NP0005, Thermo Fisher Scientific). Each membrane was blocked for $120 \mathrm{~min}$ at room temperature with $5 \%$ BSA in TBS. Both P-NKCC antibodies were pre-incubated for $30 \mathrm{~min}$ with $10 \mu \mathrm{g} / \mathrm{ml}$ non-phospho peptide. After washing, blots were incubated for $90 \mathrm{~min}$ at room temperature with secondary antibodies (\#925-68071, anti-rabbit; \#925-32214, anti-goat/sheep; both at 1:15,000) from Li-Cor Biosciences. After washing, all blots were scanned with the Odyssey CLx Imaging System (Li-Cor Biosciences). The band intensity was automatically determined by the accompanying software Image Studio v.5.2 (Li-Cor Biosciences).

\section{Co-IPs from muscle membranes}

For each IP from muscle lysates, $15 \mu \mathrm{g}$ of antibody was used per $50 \mu \mathrm{l}$ of Dynabeads Protein A (ThermoFisher Scientific; \#10001D). Protein A-bound IgG was crosslinked using $20 \mathrm{mM}$ dimethylpimelimidate (DMP, Pierce/ ThermoFisher Scientific), as previously described [35]. The Rx membrane lysate was diluted with an equal volume of Rx buffer without KCL or ATP (25 mM MOPS, $\mathrm{pH} 7.5,0.1 \mathrm{M} \mathrm{NaCl}, 10 \mathrm{mM} \mathrm{MgCl}_{2}, 60 \mathrm{mM}$ octylglucopyranoside, $0.5 \%$ IGEPAL CA-630, $10 \%$ glycerol), and the RIPA1 extract was diluted to $750 \mu \mathrm{l}$ using RIPA1 buffer. Both the diluted Rx and RIPA1 lysates were precleared with $20 \mu \mathrm{l}$ of Dynabeads Protein A (pre-rinsed in Rx or RIPA1 buffer) for $30 \mathrm{~min}$ at $4{ }^{\circ} \mathrm{C}$. A $100-\mu \mathrm{l}$ aliquot was removed as "Input" before proceeding with each IP. The lysates were divided evenly among the IP antibodies $(200 \mu \mathrm{l}$ each for RIPA1, $300 \mu \mathrm{l}$ each for Rx), and incubated at $4{ }^{\circ} \mathrm{C}$ for $2 \mathrm{~h}$ with gentle rotation. Dynabeads were collected magnetically, and the Unbound supernatants were transferred to a fresh tube. The beads were rinsed 5 times with $500 \mu \mathrm{l}$ of ice-cold $0.5 \times$ TBST $(5 \mathrm{mM}$ Tris, $\mathrm{pH} 7.5$, $83 \mathrm{mM} \mathrm{NaCl}, 0.05 \%$ Tween-20); beads were transferred to a fresh tube at the second rinse. Immunoprecipitated proteins were eluted under non-reducing conditions in Laemmli sample buffer lacking dithiothreitol (DTT) [37], and the eluate was transferred to a fresh tube.

\section{In-gel protein digestion and LC-MS/MS analysis}

IPs and analyses were performed using triplicate biological samples for each antibody and muscle genotype. Gastrocnemius muscles containing the SC were from C57BL/6 (WT) mice and a new mouse strain homozygous for the genetic ablation of a large $5^{\prime}$ coding exon in the Svil gene $\left(\mathrm{Svil}^{-/-}\right)$. Negative controls were gastrocnemius muscles from $\mathrm{Sgcg}^{-/-}$mice [16] and IPs with nonspecific rabbit IgG. DTT (final concentration of $2 \mathrm{mM}$ ) was added to the immunoprecipitated samples before they were electrophoresed into a $10 \%$ Laemmli resolving gel [37], without a stacking gel, until the dye front was $\sim 1 \mathrm{~cm}$ below the bottom of the loading wells; at least two blank wells separated samples. Gel proteins were then visualized with the Invitrogen Novex Colloidal Blue Staining Kit, according to the manufacturer's protocol (ThermoFisher Scientific; \#LC6025). Each IP sample was excised as a single gel piece. Gel pieces were subjected to in-gel trypsin digestion after reduction with DTT and alkylation with iodoacetamide. Peptides eluted from the gel were lyophilized and re-suspended in $25 \mu \mathrm{l}$ of $5 \%$ acetonitrile with $0.1 \%(\mathrm{v} / \mathrm{v})$ trifluoroacetic acid (TFA). Using an injection volume of $3 \mu \mathrm{l}$, peptides were loaded by a Waters nanoAcquity UPLC (Waters Corp., Milford, $\mathrm{MA})$ in $5 \%$ acetonitrile $(0.1 \%$ formic acid $(\mathrm{v} / \mathrm{v}))$ at 4.0 $\mu \mathrm{l} / \mathrm{min}$ for $4.0 \mathrm{~min}$ to a $100 \mu \mathrm{m}$ I.D. fused-silica pre-column (Kasil frit) packed with $2 \mathrm{~cm}$ of $5 \mu \mathrm{m}$ (200Å) Magic C18AQ (Bruker-Michrom, Billerica, MA). Peptides were then eluted at $300 \mathrm{nl} / \mathrm{min}$ via a $75-\mu \mathrm{m}$ I.D. gravity-pulled analytical column packed with $25 \mathrm{~cm}$ of $3 \mu \mathrm{m}$ (100A) Magic C18AQ using a linear gradient from 5-35\%B (mobile phase $\mathrm{A}$, water $+0.1 \%(\mathrm{v} / \mathrm{v})$ formic acid; mobile phase $\mathrm{B}$, acetonitrile $+0.1 \%(\mathrm{v} / \mathrm{v})$ formic acid) over 90 $\mathrm{min}$. Ions were introduced by positive electrospray ionization via liquid junction electrode into a Thermo Scientific Q Exactive hybrid mass spectrometer. Mass spectra were acquired over $m / z 300-1750$ at 70,000 resolution $(m / z 200)$ using an AGC (automatic gain control) target ion population of 1e6. Data-dependent acquisition selected the top 10 most abundant precursor ions for tandem mass spectrometry using higher-energy C-trap dissociation (HCD) using an isolation width of $1.6 \mathrm{Da}$, a normalized collision energy of 27 , a maximum ion fill time of $110 \mathrm{~ms}$, and an AGC target ion population of $1 \mathrm{e} 5$. Tandem mass spectra were acquired at $17,500(\mathrm{~m} / z$ 200) resolution.

\section{Proteomics data analysis}

Raw data files were peak processed with Proteome Discoverer (version 1.4, Thermo Scientific) followed by identification using Mascot (version 2.5, Matrix Science, Boston, MA) against the SwissProt Mus musculus database (download 04/2018). Search parameters included full trypsin specificity with up to 2 missed cleavages, fixed modification of cysteine carbamidomethylation, and variable modifications of methionine oxidation, glutamine to pyroglutamic acid conversion, and protein $\mathrm{N}$-terminal acetylation. Assignments were made using a $10 \mathrm{ppm}$ mass tolerance for the precursor and $0.05 \mathrm{Da}$ mass tolerance for the fragments. All non-filtered search results were processed by Scaffold software (version 4.4.4, Proteome Software, Inc., Portland. OR), utilizing the Trans-Proteomic Pipeline 
(Institute for Systems Biology, Seattle, WA). Rx extracts and RIPA1 extracts were analyzed separately. For both extracts, threshold values were set at $80 \%$ for peptides (false-discovery rates (FDR) of $0.45 \%$ for Rx extract and $0.53 \%$ for RIPA extracts) and $99 \%$ for proteins (2 peptide minimum; Rx extracts, FDR 20.0\%; RIPA extracts, FDR 12.0\%). Quantitative comparisons were made in the Scaffold software, using normalized weighted spectra, with an ANOVA significance level $P<0.05$ without a post-test. Application of a Benjamini-Hochberg multiple test correction eliminated as significant all of the $\mathrm{Rx}$ buffer interactors and $\beta$-sarcoglycan and all proteins with higher $P$ values in RIPA1 buffer, but the overall $P$ values were unchanged. Interactors were listed in order of increasing $P$ value from both Rx (Additional file 3, Table S2) and RIPA (Additional file 4, Table S3) extracts and edited manually for candidate Sgcg interactors, as described below.

Proteins specifically co-immunoprecipitating with Sgcg were defined as those that had significantly higher total normalized weighted spectral counts in IPs from C57BL/6 wild-type muscles and/or from Svil-targeted $\left(\mathrm{Svil}^{-/-}\right)$muscles, as compared to spectral counts from $\mathrm{Sgcg}^{-/-}$muscles and spectral counts in IPs with control IgG from any muscle type. Spectral counts were normalized based on each protein's predicted molecular mass. "Top candidate interactors" were represented by $\geq 3$ total normalized spectral counts from WT or Svil ${ }^{-/-}$ muscles and were selected based on $P$ values $<0.05$ and quantitative profiles that showed increases of total normalized spectral counts that were $\geq 2$-fold over those in each of the two types of negative controls. In most cases, total normalized spectral counts were greatly reduced in $\mathrm{Sgcg}^{-/-}$muscles and very few counts were observed with the IgG control antibody. "Other candidate interactors" also had $P$ values $<0.05$, but were below the 2 -fold spectral count threshold or were elevated only in $\mathrm{Svil}^{-1-}$ muscles. Two proteins (myotilin, titin) made this cut-off when the high background counts observed with control IgG were subtracted first. Because titin and myotilin mutations are responsible for LGMD, type 2J and type 1A, respectively [50], we drew the cut-off for statistical significance at $P<0.05$ under these conditions. Exceptions were made for $\alpha$ - and $\beta$-sarcoglycans, known to be part of the SC [51], and for candidate PP1 $\beta$-binding interactors close to the arbitrary 2-fold cut-off. A group of F-actinbinding proteins, e.g., spectrin, dystrophin, and filamin $\mathrm{C}$, were recovered at approximately equal abundance from all three muscle types with anti-Sgcg, but not with control IgG, and were not considered further (Additional files 3 and 4; Tables S2 and S3).

This experimental approach may be inherently limited by binding of residual actin filaments to IgG [52], leading to high backgrounds of F-actin-binding proteins. Co-IPs also were performed with affinity-purified antibodies against the actin-binding protein archvillin (anti-mAV) and Rx and RIPA1 extracts of WT muscle and muscles deficient in archvillin. No archvillin-associated candidate interactors were identified because of high background binding with rabbit IgG.

Direct and indirect structural and signaling relationships among the candidate Sgcg interactors were identified using the Connect command in Ingenuity Pathway Analysis (IPA) Path Designer software, version 01-14 (Qiagen Bioinformatics, Redwood City, CA) Sgcg interactions with Svil [26] and NKCC1 were added manually and positioned with the IPA Auto-Layout command. The B-Raf, MEK1/2, and ERK1/2 signaling cascade was added manually, along with their connections to sarcolemmal proteins. Interactor colors and designations were manipulated for clarity using Adobe Photoshop CS6 (Adobe Systems, Inc.).

\section{Plasmids}

Elizabeth McNally (Northwestern University Feinberg School of Medicine, Chicago, IL) generously provided the plasmid encoding untagged murine Sgcg [36]. Myc-DDK (Flag)-tagged murine $\beta$ - (\#MR204617), $\delta$ - (\#MR221060) and $\gamma^{-}$(\#MR223013) sarcoglycan cDNAs were from OriGene Technologies (Rockville, MD), as were MycDDK-tagged cDNAs encoding mouse Ppp1r12b/MYPT2 (\#MR226968) and mouse tensin 2 (tensin like C1 domaincontaining phosphatase, isoform 1/Tenc1; \#MR211954). The plasmid encoding EGFP-PP $1 \beta / \delta$ (EGFP-C1 vector) was obtained from Mathieu Bollen, (KU Leuven, Leuven, Belgium), via A. J. Baucum (Indiana University-Purdue University, Indianapolis, IN) [53]. Plasmid encoding human PP1 $\beta$-EGFP (EGFP-N3 vector) was a gift from Angus Lamond and Laura Trinkle-Mulcahy (Addgene plasmid \#44223; http://n2t.net/addgene:44223; RRID:Addgene_44223) [54]. EGFP-tensin2 (human) was a gift from David Critchley and Kenneth Yamada (Addgene plasmid \#105298; http://n2t.net/addgene: 105298; RRID:Addgene_105298) [55]. HA-tagged human NKCC1 (pdDNA3.1-HA-CFP-hNKCC1 WT (NT15-H)) was a gift from Biff Forbush (Addgene plasmid \#49077; http://n2t.net/addgene:49077; $\quad$ RRID:Addgene_49077 [56]).

GST-bSV1398-1792 was described previously [57]. A pIRES2-EGFP vector containing full-length human Sgcg cloned between the EcoRI and BamHI sites [58] was used as a template to create plasmids encoding only the $35 \mathrm{~N}$-terminal residues fused with $\mathrm{C}$-terminal EGFP. The vector was first modified to contain an internal BamHI site by converting Leu-36 to glycine and Tyr-37 to serine using the QuikChange Site-Directed 
Mutagenesis kit (Agilent Technologies; \#200519) and the L36Y37-BamHI-sense and L36Y37-BamHI-antisense primers in Additional file 1, Table S1. The modified vector was restriction digested with BamHI and NotI, and the similarly digested EGFP cassette from pEGFP-N3 was directionally ligated to be in-frame with the Sgcg cytoplasmic domain, i.e., residues 1-35. The Sgcg1-35-EGFP vector was then used as a template to generate hSgcg-135-EGFP fragments to clone into either the pET30a vector for His-tagging using the Sgcg-BglII-start-For and Sgcg-EcoRI-end-Rev primer pair or into the pMALc5x vector (New England BioLabs, Beverly, MA; \#N8108S) for maltose-binding protein tagging using the Sgcg-ForEcoRV-1Met and Scgc-EcoRI-end-Rev primer pair. EGFP only controls were cloned into the same vectors using a BamHI-NotI double digest from pEGFPN3 into pET30a, and an XmnI-EcoRI double digest from pET30a-EGFP into pMALc5x. Vectors containing wild-type hSgcg-135 -EGFP were then mutated at Tyr-6 to Ala using the QuikChange mutagenesis kit and the Scgc-Y6A-sense and antisense primer pair. All restriction enzymes were from New England BioLabs (Beverly, MA).

The plasmid encoding 3X-HA-tagged human NKCC1 synthetic cDNA with convenient restriction sites [56] was modified in two ways. First, we eliminated the sequence encoding all of the transmembrane domains and much of the cytoplasmic domains with a double restriction digest using $H p a I$ and $E c o R V$. We then re-ligated the blunt ends to generate 3xHA-CFP-hNKCC1-cyto, which encodes the 3xHA-CFP tag, followed by the cytosolic NKCC1 N-terminal amino acids Glu-2 through Val-141 and the 91 residues of the cytosolic NKCC1 C-terminus (I-1118 through S-1196) [56]. Second, we generated a plasmid encoding a control 3XHA-CFP protein, which lacks the entire NKCC1 sequence, by inserting a TAA stop codon immediately downstream of the CFP-coding sequence using PCR with the QuikChange ${ }^{\circledR}$ II XL site-directed mutagenesis kit (Agilent Technologies; \#200521) and primers HA-CFP-Stp For and HA-CFP-Stp Rev (Additional file 1, Table S1). All plasmids were verified by end sequencing.

\section{Cell culture, screens with exogenously expressed proteins, and proximity ligation assay}

Human RH30 rhabdomyosarcoma cells (SJC-RH30, American Type Culture Collection \#CRL-2061, Manassas, VA) were maintained at $37{ }^{\circ} \mathrm{C}$ and $5 \% \mathrm{CO}_{2}$ in RPMI-1640 medium modified to contain $2 \mathrm{mM}$ L-glutamine, $10 \mathrm{mM}$ HEPES, $1 \mathrm{mM}$ sodium pyruvate, 4500 $\mathrm{mg} / \mathrm{L}$ glucose, $1.5 \mathrm{~g} / \mathrm{L}$ sodium bicarbonate (\#A1049101, Thermo Fisher Scientific), supplemented with 10\% heat-inactivated fetal bovine serum, an additional 2
mM L-glutamine, and Pen-Strep. Transfections were done in $6-\mathrm{cm}$ dishes that had been plated the day before so that the cells would be $\leq 60 \%$ confluent, using Lipofectamine 2000 (Thermo Fisher Scientific) and a scaled-down version of the manufacturer's instructions. First, an equimolar mixture of C-terminally myc-DDKtagged $\beta$-- $\delta$-- and $\gamma$-sarcoglycan cDNAs was made in water at a total concentration of $0.3 \mu \mathrm{g} / \mu \mathrm{l}$. For co-transfections in a $6-\mathrm{cm}$ dish, with or without coverslips, $5 \mu \mathrm{l}$ of this mix $(1.5 \mu \mathrm{g})$ or $1.5 \mu \mathrm{g}$ of an empty Flag vector control were then mixed with $1.5 \mu \mathrm{g}$ of the other mammalian expression plasmid before dilution to $250 \mu \mathrm{l}$ in OptiMEM reduced-serum medium (\#31985, Thermo Fisher Scientific). Concurrently, $10 \mu \mathrm{l}$ of Lipofectamine 2000 per transfection were diluted to $250 \mu \mathrm{l}$ in OptiMEM. Both solutions were incubated for $5 \mathrm{~min}$ at room temperature and then combined (500 $\mu \mathrm{l}$ per transfection dish) and incubated at room temperature for an additional $10 \mathrm{~min}$. The culture medium in each plate was replaced with $4.5 \mathrm{ml}$ of fresh medium immediately before the plasmid DNA complexes in OptiMEM were added drop-wise across each plate. Plates were then incubated for 3 to $4 \mathrm{~h}$ at $37^{\circ} \mathrm{C}$ and $5 \% \mathrm{CO}_{2}$ before the medium was replaced with $5 \mathrm{ml}$ of fresh growth medium and the incubation was continued overnight. Cells on coverslips in 6-well plates were cultured and transfected the same way, except using half the amounts of plasmid DNA and Lipofectamine 2000 in $100 \mu \mathrm{l}$ of OptiMEM and a total volume of $2 \mathrm{ml}$ medium per well. Cells were harvested 23 to $25 \mathrm{~h}$ post-transfection for either co-IP or fixation for immunofluorescence or proximity ligation assay (PLA). Fixation was performed as previously described [59], using either ice-cold methanol for $15 \mathrm{~min}$ or $4 \%$ paraformaldehyde in CSK buffer (10 mM PIPES, pH 6.8, $300 \mathrm{mM}$ sucrose, $100 \mathrm{mM}$ sodium chloride, $3 \mathrm{mM}$ magnesium chloride, $1 \mathrm{mM}$ EGTA [60]) for $30 \mathrm{~min}$ on ice. Pre-extraction experiments involved treating cells on coverslips with $0.1 \%$ Triton X-100 in CSK buffer for $4 \mathrm{~min}$ on ice and rinsing briefly with PBS before fixation and immunofluorescence imaging, as described above.

PLA was performed using the Duolink In Situ Orange kit (Sigma \#DUO92102), according to the manufacturer's instructions, with mouse anti-HA (Cell Signaling \#2367, 1:200) and rabbit anti-Sgcg (Proteintech \#18102AP, 1:200) as primary antibodies. PLA speckles were counted by hand after signal inversion and $\sim 3$-fold enlargement using Adobe Photoshop. Nuclei associated with zero speckles were assumed to be untransfected cells. Graphical and statistical analyses were performed with GraphPad Prism. 


\section{Co-IPs and supernatant-depletion assays}

Standard co-IP experiments and supernatant-depletion assays were carried out after extraction of RH30 cells with modified RIPA1 (no SDS). Cells between passage 5 and 28 in 6-cm dishes were transfected for these experiments as follows: (1) with a mixture of Myc-DDK (Flag)tagged murine $\beta-, \delta-, \gamma$-sarcoglycans and either HA-CFP, HA-CFP-Cyto, or HA-CFP-NKCC1; or (2) with MycFlag-tagged sarcoglycans, or an empty Flag vector, and either PP1 $\beta$-EGFP, EGFP- PP1 $\beta$ or EGFP. Transfected cells were grown for $24 \mathrm{~h}$ and were $50-60 \%$ confluent when extracted on ice for $15 \mathrm{~min}$ in $350 \mu \mathrm{l}$ of pre-chilled modified RIPA1 buffer (no SDS), plus protease and phosphatase inhibitors (\# P8340, P2850, P5726, SigmaAldrich). Extracts were collected by scraping and transferred to $1.5-\mathrm{ml}$ polypropylene centrifuge tubes, sonicated for 25 pulses with a Branson sonifier, as above, and centrifuged at $18,000 \times g$ for $10 \mathrm{~min}$ at $4{ }^{\circ} \mathrm{C}$. The resulting supernatants were removed to a fresh $1.5-\mathrm{ml}$ tube and used for either co-IPs or supernatant-depletion assays.

For experiments with HA-tagged proteins in RH30 cell lysates, Protein A Dynabeads were incubated for $1 \mathrm{~h}$ at room temperature either with monoclonal rabbit antiHA antibody, clone C29F4, (for HA-CFP-NKCC1 experiments), or with rabbit polyclonal anti-Flag. Beads were rinsed once with RIPA1 buffer and split into equal fractions. The rinse was removed from each tube, and the beads were resuspended in $300 \mu \mathrm{l}$ of each cell extract and incubated with rotation for $1 \mathrm{~h}$ at $4{ }^{\circ} \mathrm{C}$. For experiments involving EGFP, $25 \mu \mathrm{l}$ of GFP-Trap Dynabeads (product code gtd, Chromotek Inc., Islandia, NY, USA) were used per IP and washed once with ice-cold RIPA1 buffer before the addition of cell extract.

Co-IPs and supernatant-depletion assays were carried out similarly, except co-IPs focused on the amounts of proteins in pellets ("Bound") and supernatant-depletion assays focused on the differences in protein concentration before ("Input") and after ("Unbound") centrifugation. The latter approach better detects low-avidity interactions [61]. In supernatant-depletion assays, either $20 \mu \mathrm{l}$ of beads with bound anti-HA antibody or $25 \mu \mathrm{l}$ of GFP-Trap Dynabeads were mixed with $300 \mu$ of each cell extract for $1 \mathrm{~h}$ at $4{ }^{\circ} \mathrm{C}$. A $100-\mu \mathrm{l}$ "Input" sample was taken, and the remainder of each mixture was quickly sedimented through a cushion of $20 \%$ sucrose in RIPA1 buffer using narrow-bore BioRad tubes (\#223-9502) and a swinging-bucket rotor at $800 \times g$ for $5 \mathrm{~min}$. The top $100 \mu \mathrm{l}$ was taken as the "Unbound" sample. Input and Unbound samples were resolved on $12 \%$ SDS-polyacrylamide gels and electroblotted.

Supernatant-depletion assays [61] also were carried out with purified, recombinant $\mathrm{N}$-terminally tagged archvillin C-terminus and C-terminally tagged Sgcg cytoplasmic domain. The GST-bSV1398-1792 protein is respectively $94.43 \%$ and $96.96 \%$ identical to the corresponding sequences in murine and human archvillins (from Clustal2.1, Conway Institute UCD, Dublin, Ireland), and was made as described previously [57]. All recombinant EGFP proteins were made in Rosetta 2 pLyS bacteria (\#71400, Novagen/Sigma-Aldrich), as described for antigen production. His-EGFP-tagged proteins were purified via NiNTA agarose (Qiagen), and MBP-tagged proteins were purified via amylose (New England BioLabs), according to the manufacturers' instructions. We removed the tags from both sets of proteins (thrombin digestion for His tags, Factor Xa proteolysis for MBP), and re-purified them with NiNTA agarose or by ion-exchange chromatography, respectively. GST-bSV-1398-1792 was mixed with the EGFP proteins in a total volume of $250 \mu \mathrm{l}$ and incubated for $30 \mathrm{~min}$ at $4{ }^{\circ} \mathrm{C}$ with rotation before the addition of $25 \mu \mathrm{l}$ of glutathione-Sepharose beads (\#170756-01, GE Healthcare). After an additional 30-min incubation, $100 \mu \mathrm{l}$ of the total slurry was removed as the "Input" sample; the rest was loaded atop a $25 \%$ sucrose cushion in a narrow diameter tube and centrifuged, as described above. Input and Unbound samples were resolved on 12\% SDS-polyacrylamide gels and electroblotted.

\section{Muscle immunostaining}

To determine the localization of NKCC1 in EDL muscles, $10-\mu \mathrm{m}$ cryosections were obtained from EDL muscles of $\mathrm{C} 57$ and $\mathrm{Sgcg}^{-/-}$mice. Both longitudinal and cross sections were utilized. Sections were washed thrice in PBS, 10 min per wash. Sections were fixed for $5 \mathrm{~min}$ in $4 \%$ paraformaldehyde in PBS, followed by permeabilization with $0.5 \%$ Triton-X, PBS. Following blocking in 5\% bovine serum albumin (BSA) in PBS for $1 \mathrm{~h}$ at room temperature, sections were incubated overnight at $4{ }^{\circ} \mathrm{C}$ with primary antibodies diluted in $5 \%$ BSA against dystrophin (MANDYS1, 3B7) [39] and NKCC1 (1:500 NKCC1, rabbit pAb 13884-1-AP; Proteintech) or with $5 \%$ BSA alone as a negative control. Sections were then washed thrice in PBS, $10 \mathrm{~min} /$ wash, and incubated $1 \mathrm{~h}$ at room temperature in the dark with secondary antibodies diluted in 5\% BSA (1:10,000, Alexafluor 488 IgG anti-mouse (\#A11029); 1:1000 Alexafluor 568 IgG anti-rabbit (\#A11036) (Invitrogen). Sections were washed again in the dark (PBS 3 times, 10 min each), air-dried, and covered with a mounting agent (ProLongTM Diamond Antifade with Dapi, Cat\#P36962, Thermo Fisher Scientific) and coverslip. 


\section{NKCC1 inhibition in vivo}

EDL muscles from C57 or $\mathrm{Sgcg}^{-/-}$mice (9-14 wks old) were isolated and prepared for muscle mechanics as previously described [62]. EDLs with suture loops were mounted to the 800A in vitro Muscle Apparatus (Aurora Scientific, Ontario, CAN) via a rigid hook and hook to a servomotor arm. The muscles were positioned between two platinum plate electrodes, and in a Radnoti vessel filled with Ringer's solution equilibrated with $95 \% \mathrm{O}_{2} / 5 \%$ $\mathrm{CO}_{2}$ and maintained at $22{ }^{\circ} \mathrm{C}$. EDL stimulation was controlled and carried out using Aurora Scientific (Ontario, CAN) hardware and software (i.e., High Power Bi-Phase Current Stimulator, 300C Dual-Motor Level System, Digital Controller Interface, and DMC v5.420). Following determination of optimal length (Lo), muscles were subjected to three bouts of supramaximal stimulation at 150 $\mathrm{Hz}$ for $500 \mathrm{~ms}$, separated by $3 \mathrm{~min}$ to obtain maximum isometric force (Max Force). After completion of initial stimuli, EDLs were treated with bumetanide dissolved in $100 \%$ ethanol (final concentration $50 \mu \mathrm{M}$ bumetanide, $1 \%$ ethanol) or ethanol vehicle alone (final concentration $1 \%$ ethanol) for 20-30 min. A second series of Max Force measurements were conducted to determine potential changes in twitch and tetanic forces after treatment. After a 5-min rest period, EDLs underwent five eccentric contractions, consisting of a $10 \%$ Lo lengthening during the final $200 \mathrm{~ms}$ of stimulation, with a 5-min rest interval between stimuli. A 30-min wait period followed muscle mechanics. EDLs were then lightly dried, weighed, snap frozen, and stored at $-80{ }^{\circ} \mathrm{C}$. For those EDLs remaining at rest, they were pinned loosely in a petri dish with oxygenated Ringers solution containing either $50 \mu \mathrm{M}$ bumetanide, $1 \%$ ethanol, or neither substance for $30 \mathrm{~min}$. Similarly, non-mechanics EDLs were snap frozen afterwards and stored at $-80{ }^{\circ} \mathrm{C}$ for future biochemical and histological analyses.

\section{P/T-ERK1/2 and P/T-NKCC1 activation assays}

EDL and TA muscles were powdered using a mortar and pestle in dry ice and homogenized in RIPA2 buffer supplemented with PMSF and inhibitors of proteases (P8340; Sigma-Aldrich) and phosphatases (P5726; Sigma-Aldrich). Homogenates were incubated on ice for $60 \mathrm{~min}$ and centrifuged at $15,000 \times g$ for $15 \mathrm{~min}$. Protein quantifications of muscle lysates were determined by the Bradford assay (\#1863028; Thermo Fisher Scientific). For P/T-ERK1/2 analyses, EDL lysates (20 $\mu \mathrm{g}$ protein) were loaded in 4-12\% Bis-Tris Midi Protein Gels (\#WG1402A, Thermo Fisher Scientific) and electrotransferred to nitrocellulose. Each membrane was blocked for $90 \mathrm{~min}$ at room temperature with 5\% BSA (BP9703100, Thermo Fisher Scientific) in TBS. Blots were incubated with anti-phospho-ERK1/2 and anti-total-ERK1/2 primary antibodies overnight at $4{ }^{\circ} \mathrm{C}$, washed and incubated for $90 \mathrm{~min}$ at room temperature with secondary antibodies (Li-Cor Biosciences).

For P/T-NKCC1 assays, TA lysates (20 $\mu \mathrm{g}$ protein) were loaded in 3-8\% Bis-Tris Gel 1.0 mm (WG1002BOX; Thermo Fisher Scientific) and transferred to ImmobilonFL PVDF, 0.45- $\mu \mathrm{m}$ porosity (IPFL00010, EMD-Millipore) for $16 \mathrm{~h}$ at $40 \mathrm{~mA}$ in $10 \%$ methanol, $0.01 \%$ SDS, $0.1 \%$ NuPage Antioxidant (NP0005, Thermo Fisher Scientific). Each membrane was blocked for $120 \mathrm{~min}$ at room temperature with 5\% BSA in TBS. Both P-NKCC antibodies were pre-incubated for $30 \mathrm{~min}$ with $10 \mu \mathrm{g} / \mathrm{ml}$ non-phospho peptide. After washing, blots were incubated for $90 \mathrm{~min}$ at room temperature with secondary antibodies (\#925-68071, anti-rabbit; \#925-32214, anti-goat/sheep; both at 1:15,000) from Li-Cor Biosciences. After washing, all blots were scanned with the Odyssey CLx Imaging System (Li-Cor Biosciences). The band intensity was automatically determined by the accompanying software Image Studio v.5.2 (Li-Cor Biosciences).

\section{Results}

\section{Identification of new candidate sarcoglycan interactors}

To complement previous two-hybrid screens for the identification of sarcoglycan interactors [25, 26], we used a new co-immunoprecipitation (co-IP) and labelfree proteomic approach to identify proteins directly or indirectly associated with Sgcg (Fig. 1). Eliminating time-consuming ultracentrifugation and carbohydratecrosslinking lectins [46, 47], we modified the method of Yamamoto et al. [43] to enrich sarcolemmal proteins from a single murine muscle $(50 \mathrm{mg}$ to $100 \mathrm{mg}$ wet mass), using bench-top equipment available in most research laboratories. Our method avoids potentially false associations due to carbohydrate agglutination by an extracellular lectin, but sacrifices the high sarcolemmal purity of previous approaches. Our relatively rapid approach takes less than $3 \mathrm{~h}$ for up to 4 samples from individual mouse muscles (Fig. 1). Sgcg-associated proteins were interrogated after extraction of relatively low-density membranes with a cytoskeletal disruption and relaxation ( $\mathrm{Rx}$ ) buffer and after extraction of higher-density, cytoskeleton-associated fractions with an SDS-containing RIPA1 buffer (Figs. 1 and 2). To our knowledge, the higher-density, cytoskeleton-associated fractions have not been explored for sarcoglycan- or other DGC-associated proteins. Each detergent supernatant contained the sarcolemmal marker proteins dystrophin, archvillin, and Sgcg (Fig. 2B-D, lanes 6, 8). The RIPA1 extract was enriched in proteins associated with the DGC (dystrophin, archvillin, Sgcg, Sgca, $\beta$-dystroglycan) (Fig. 2B-E), and proteins associated with the sarcoplasmic reticulum (SERCA1) and 


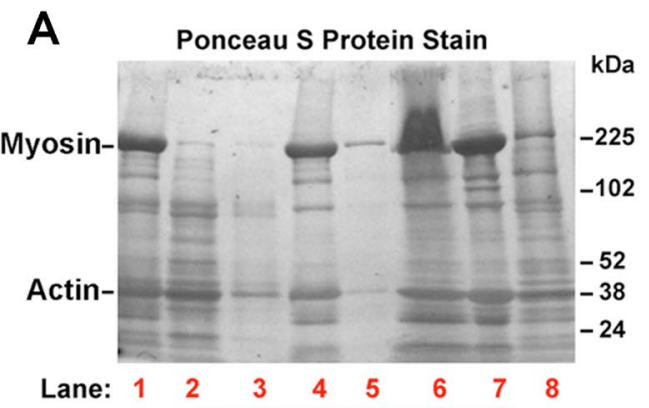

B

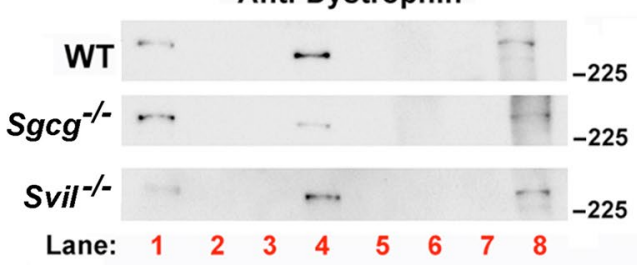

C

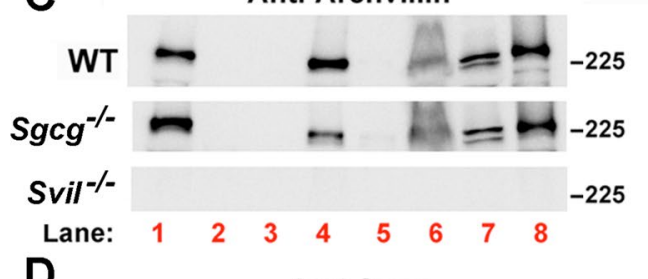

D

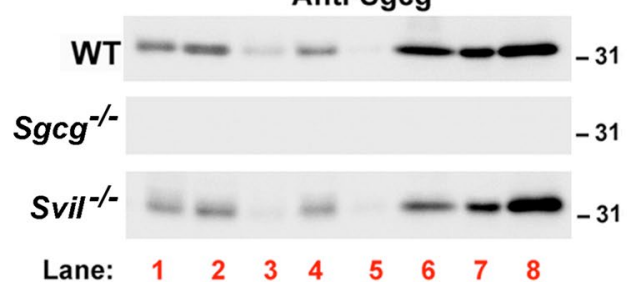

E
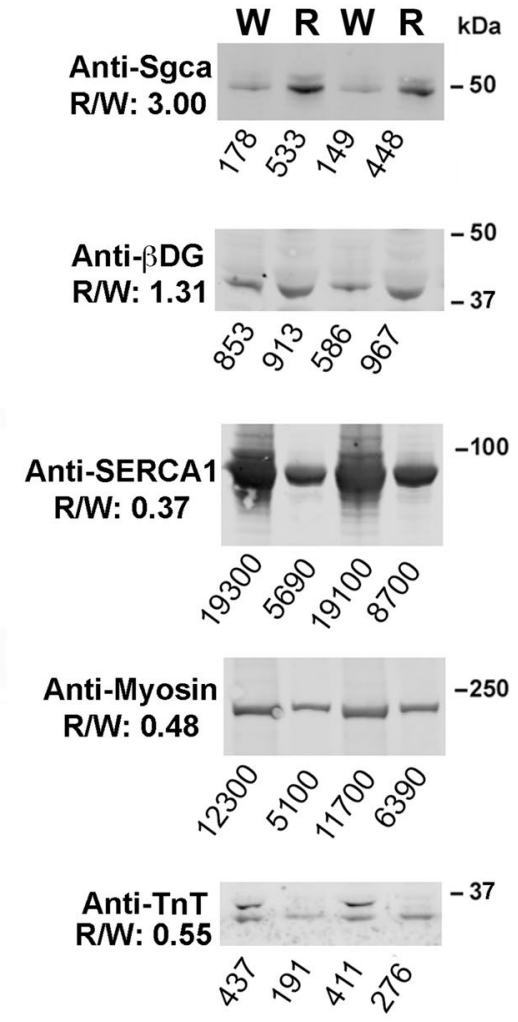

Fig. 2 Sarcolemmal proteins recovered from Rx (lane 6) and RIPA1 (lane 8) buffers. Gastrocnemius muscles ( 100 mg) from either C57BL/6 (WT)

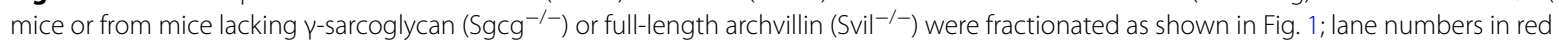
correspond with Fig. 1. Blots were stained for $\mathbf{A}$ protein with Ponceau $\mathbf{S}$ and probed for the sarcolemmal proteins $\mathbf{B}$ dystrophin, $\mathbf{C}$ archvillin, and D -sarcoglycan (Sgcg, using our new antibody). Much actin is removed by the low-salt in buffer 1 (A, lanes 2-3); most myosin II is extracted by the high salt in Rx buffer (A, lane 6) or remains insoluble in RIPA1 buffer (A, lane 7). Blots are representative of 3 biological replicates per genotype. Fractions 1-6 are normalized to total protein; fractions 7-8 are normalized by volume to each other; total protein in fraction 8 approximates that of fraction 1. Lane 1, whole muscle extracts. Lanes 2-3, discarded washes. Lane 4, enriched membranes before extraction. Lane 5, discarded Rx pellet. Lane 6, Rx supernatants. Lane 7, discarded, insoluble RIPA1 pellets. Lane 8, RIPA1 supernatants. E Comparison of protein abundance by immunoblotting of whole tissue lysates (W) vs. RIPA1 lysates (R). Equal protein loading (based on BCA quantification) for each condition was used. DGC proteins Sgca and $\beta$-dystroglycan ( $\beta$-DG) were enriched in RIPA1 extracts of wild-type C57BL/6 muscles (equivalent to lane 8 in A-D), as compared with whole muscle lysates (equivalent to lane 1 in $\mathbf{A}-\mathbf{D}$ ), whereas the sarcoplasmic reticulum marker SERCA1 and the contractile apparatus proteins myosin II and fast troponin $\mathrm{T}(\mathrm{TnT})$ were depleted. Mean band intensities from ImageStudio software are indicated below each lane and used to calculate the ratios of RIPA1 to whole muscle lysate (R/W) shown to the left of each blot

contractile apparatus (actin, myosin II, troponin-T) were reduced (Fig. 2A, E). The Rx supernatants contained more and larger particulates than did the RIPA supernatants, but no large membrane sheets remained (Additional file 5, Fig. S2).

We identified new candidate direct or indirect interaction partners for the SC by comparing proteins recovered using our new high-avidity anti-Sgcg antibody crosslinked onto magnetic beads with those obtained from negative controls (Fig. 3; Additional files 3, and 4; Tables S2 and S3). Gastrocnemius muscles containing the SC were from C57BL/6 (WT) mice and a new mouse strain homozygous for the genetic ablation of a large $5^{\prime}$ coding exon in the Svil gene 


\begin{tabular}{|c|c|c|c|c|c|c|c|c|c|c|}
\hline \multirow[t]{3}{*}{$\mathbf{A}$} & \multirow{2}{*}{\multicolumn{10}{|c|}{$\begin{array}{c}\text { RX Buffer } \\
\text { Total Normalized Weighted Spectral Counts }\end{array}$}} \\
\hline & & & & & & & & & & \\
\hline & \multicolumn{4}{|c|}{$\begin{array}{l}\text { Antibody Specificity: } \\
\text { Muscle Genotype: }\end{array}$} & \multirow[t]{2}{*}{$\begin{array}{l}\text { Sgcg } \\
\text { WT }\end{array}$} & \multirow[t]{2}{*}{$\begin{array}{l}\text { Sgcg } \\
\text { Svil--/- }\end{array}$} & \multirow[t]{2}{*}{$\begin{array}{l}\text { Sgcg } \\
\text { Sgcg-/- }\end{array}$} & \multirow[t]{2}{*}{$\begin{array}{l}\mathrm{Rb} \lg G \\
\mathrm{WT}\end{array}$} & \multirow[t]{2}{*}{$\begin{array}{l}\text { Rb IgG } \\
\text { Svil-/- }\end{array}$} & \multirow[t]{2}{*}{$\begin{array}{l}\text { Rb lgG } \\
\text { Sgcg-/- }\end{array}$} \\
\hline Protein & Gene & MW (kDa) & $P(A N O V A)$ & Quant Profile & & & & & & \\
\hline PP1 $\beta$, catalytic subunit & Ppp1cb & 37 & 0.02 & 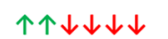 & 24 & 16 & 4 & 0 & 0 & 2 \\
\hline Gamma-sarcoglycan & Sgcg & 32 & 0.06 & & 6 & 3 & 2 & 0 & 0 & 2 \\
\hline Beta-sarcoglycan & $S g c b$ & 35 & 0.60 & & 3 & 0 & 0 & 0 & 0 & 0 \\
\hline \multirow[t]{4}{*}{ B } & & & & & \multirow{2}{*}{\multicolumn{6}{|c|}{$\begin{array}{l}\text { RIPA Buffer } \\
\text { Total Normalized Weighted Spectral Counts }\end{array}$}} \\
\hline & & & & & & & & & & \\
\hline & & & \multirow{2}{*}{\multicolumn{2}{|c|}{$\begin{array}{l}\text { Antibody Specificity: } \\
\text { Muscle Genotype: }\end{array}$}} & Sgcg & Sgcg & Sgcg & Rb Ig G & $R b \lg G$ & $R b \lg G$ \\
\hline & & & & & WT & Svil-/- & Sgcg-/- & & Svil-/- & Sgcg-/- \\
\hline Protein & Gene & $M W(k D a)$ & $P(A N O V A)$ & Quant Profile & & & & & & \\
\hline \multicolumn{11}{|l|}{ Top Candidate Interactors } \\
\hline Gamma-sarcoglycan & Sgcg & 32 & $<0.00010$ & 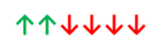 & 18 & 18 & 0 & 0 & 0 & 0 \\
\hline Delta-sarcoglycan & Sgcd & 32 & $<0.00010$ & 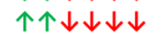 & 7 & 10 & 2 & 0 & 0 & 0 \\
\hline PP1 $\beta$, catalytic subunit & Ppp1cb & 37 & 0.00028 & 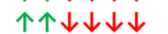 & 42 & 42 & 12 & 0 & 0 & 0 \\
\hline $\begin{array}{l}\text { Protein phosphatase } 1 \text { regulatory } \\
\text { subunit } 12 B(\text { MYPT2) }\end{array}$ & Ppp1r12b & 109 & 0.00079 & 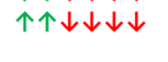 & 96 & 104 & 51 & 0 & 0 & 0 \\
\hline Microtubule-associated protein tau & Mapt & 76 & 0.0014 & 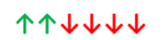 & 8 & 13 & 3 & 0 & 0 & 0 \\
\hline $\begin{array}{l}\text { Nascent polypeptide associated } \\
\text { complex subunit, alpha, muscle }\end{array}$ & Naca & 220 & 0.0021 & 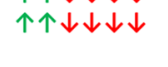 & 53 & 63 & 14 & 0 & 0 & 1 \\
\hline $\begin{array}{l}\text { Eukaryotic translation initiation } \\
\quad \text { factor } 4 \text {, gamma } 1\end{array}$ & Eif4g1 & 175 & 0.0021 & 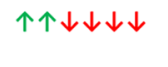 & 12 & 19 & 4 & 0 & 0 & 0 \\
\hline EGF receptor substrate 15 & Eps15 & 98 & 0.0022 & 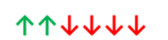 & 13 & 25 & 7 & 0 & 0 & 0 \\
\hline Beta-sarcoglycan & Sgcb & 35 & 0.0054 & $\uparrow \uparrow \downarrow \downarrow \downarrow \downarrow$ & 23 & 23 & 0 & 0 & 0 & 0 \\
\hline $\begin{array}{l}\text { Transforming acidic coiled-coil } \\
\text { containing protein } 2\end{array}$ & Tacc2 & 124 & 0.0072 & 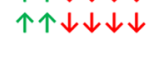 & 18 & 23 & 5 & 0 & 0 & 0 \\
\hline $\begin{array}{l}\text { Transcriptional activator protein } \\
\text { Pur-alpha }\end{array}$ & Pura & 35 & 0.0085 & 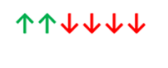 & 14 & 11 & 4 & 0 & 0 & 0 \\
\hline $\begin{array}{l}\text { Thiodoxin-dependent peroxide } \\
\text { reductase }\end{array}$ & $\operatorname{Prd} \times 3$ & 28 & 0.013 & 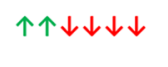 & 27 & 30 & 7 & 0 & 0 & 0 \\
\hline $\begin{array}{l}\text { Solute carrier family 12, } \\
\text { member } 2 \text { (NKCC1) }\end{array}$ & Slc12a2 & 131 & 0.016 & 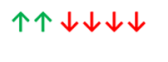 & 18 & 28 & 12 & 0 & 0 & 0 \\
\hline Nucleolin & $\mathrm{Ncl}$ & 77 & 0.031 & 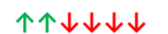 & 16 & 18 & 5 & 0 & 2 & 0 \\
\hline Alpha-sarcoglycan & Sgca & 43 & 0.098 & & 3 & 3 & 0 & 0 & 0 & 0 \\
\hline \multicolumn{11}{|l|}{ Other Candidate Interactors } \\
\hline Myotilin & Myot & 55 & 0.033 & 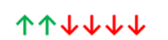 & 78 & 64 & 44 & 15 & 18 & 29 \\
\hline Titin & Ttn & 3906 & 0.048 & 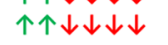 & 4700 & 4532 & 2822 & 2363 & 2866 & 2473 \\
\hline Tensin2 / TenC1 & Tns2 & 150 & 0.019 & $\downarrow \uparrow \downarrow \downarrow \downarrow \downarrow$ & 9 & 18 & 6 & 0 & 0 & 0 \\
\hline PUR6, Multifunctional protein ADE2 & Paics & 47 & 0.044 & $\downarrow \uparrow \downarrow \downarrow \downarrow \downarrow$ & 3 & 12 & 3 & 0 & 0 & 0 \\
\hline Palladin, isoform 6 & Palld & 151 & 0.046 & $\downarrow \uparrow \downarrow \downarrow \downarrow \downarrow$ & 2 & 4 & 2 & 0 & 0 & 0 \\
\hline \multicolumn{11}{|c|}{ 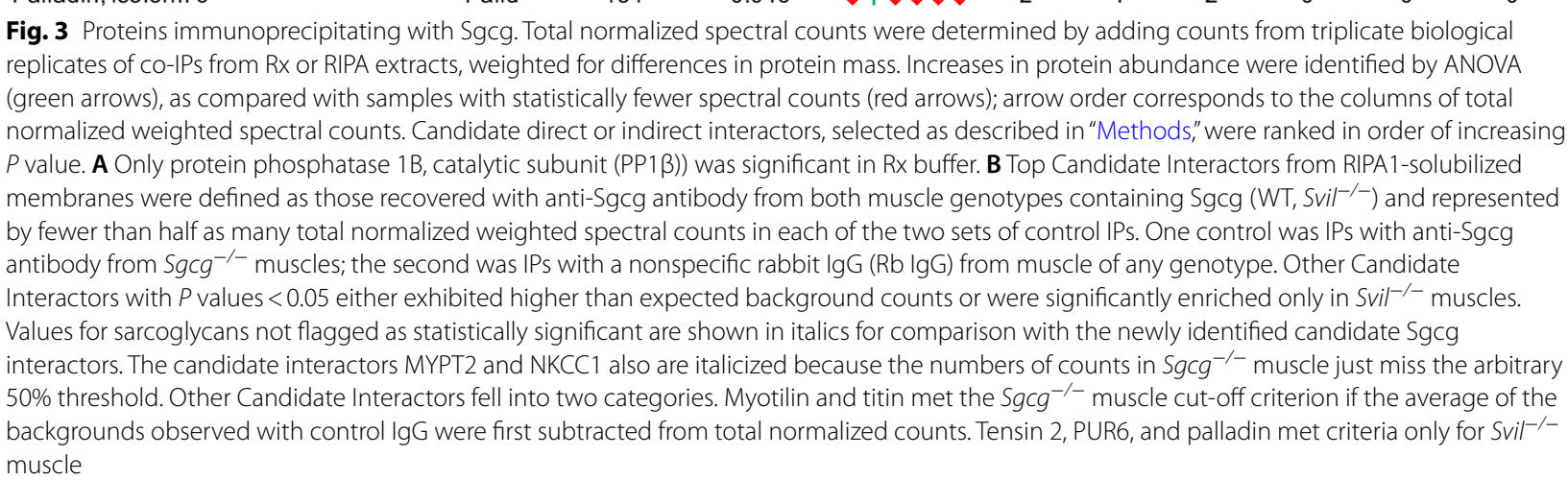 } \\
\hline
\end{tabular}

$\left(\right.$ Svil $\left.^{-/-}\right)$. Negative controls were gastrocnemius muscles from $\mathrm{Sgcg}^{-/-}$mice [16] and IPs with nonspecific rabbit IgG.
All four sarcoglycans were immunoprecipitated with anti-Sgcg from the WT and Svil ${ }^{--}$RIPA1 extracts although the $P$ value for Sgca, the least tightly 
incorporated sarcoglycan subunit [63, 64], was only 0.095 , due to a low number of specific spectral counts (Fig. 3). These results are consistent with the previous observation that the SC is stable in SDS-containing buffers [65] and suggest that the other specifically coimmunoprecipitating proteins identified here (Fig. 3) are excellent new candidate interactors for the SC.

The top co-immunoprecipitating, non-sarcoglycan protein in both Rx and RIPA1 extracts is the Ser/Thr protein phosphatase catalytic subunit $1 \mathrm{~B}(\mathrm{PP} 1 \beta$, also called PP1 $\delta$ ) (Fig. 3). Additional candidate interactors emerged from the RIPA1 extract of sarcolemmal proteins associated with large detergent-resistant structures (Fig. 3A vs. B). Because PP1 $\beta$ was the top candidate, the presence of MYPT2, NKCC1, and tensin2, which are known or predicted to bind to PP1 $\beta$, also was intriguing (Fig. 3). The $\mathrm{N}$-terminal tail of the $\mathrm{Na}^{+}-\mathrm{K}^{+}-2 \mathrm{Cl}^{-}$symporter (NKCC1, gene $S l c 12 a 2$ ) can scaffold PP1 $\beta$ and a regulatory kinase for reciprocal control of NKCC co-transport activity [66]. NKCC1 supports the opening of voltage-activated $\mathrm{Ca}^{++}$ channels by accumulating $\mathrm{Cl}^{-}$ions in the cytosol through cotransport with extracellular $\mathrm{Na}^{+}$and $\mathrm{K}^{+}$lost during active muscle contraction [67]. MYPT2, encoded by the Ppp1r12b gene, concentrates PP1 catalytic subunits along myosin II filaments $[68,69]$ and localizes near the $Z$-line and with mitochondria in cardiac muscle [70]. Tensin2 is structurally related to the focal adhesion-associated mechanosensory protein tensin1, which binds directly to both PP $1 \alpha$ and integrin to regulate cell migration and adhesion [71-73]. Thus, PP1 $\beta$ or a PP1 $\beta$-associated protein might regulate the previously observed Sgcg-mediated ERK1/2 signaling [18, 20,24], directly or indirectly, as part of a larger signaling complex.

Other candidate sarcoglycan interactors in Fig. 3B are also mechanistically plausible. While many of these candidate interactors are not an integral part of the sarcolemma, per se, they demonstrate the utility of the enrichment strategy, as they represent proteins that bridge the sarcolemma with other cellular compartments, and sites where loss of function mutations cause neuromuscular diseases. For example, mutations in myotilin and titin are causal for LGMD [50], and Sgcg binds titin sequences [25]. Palladin scaffolds the formation of cytoskeletal structures, including costameres [74, 75]. Others of these proteins are involved in membrane trafficking (Ep215, TACC2, Mapt), cellular responses to stress (Naca, Prdx3), and nuclear function (eIF4G, PURA, nucleolin).

The extractabilities of PP1 $\beta$, MYPT2, and NKCC1 into $\mathrm{Rx}$ and RIPA buffers were roughly comparable across genotypes (Additional file 6, Fig. S3). There was no apparent correlation between the amounts of these novel candidate interactors in the RIPA extracts (Additional file 6,
Fig. S3) and the numbers of normalized spectral counts obtained after co-IP with anti-Sgcg from muscle (Fig. 3), especially for glycosylated and nonglycosylated NKCC1 [76, 77] (Additional file 6, Fig. S3C). These results suggest that neither large changes in protein expression levels nor extractability account for the observed reductions in the numbers of spectral counts in co-IPs from $\mathrm{Sgcg}^{-/-}$muscle and that the reduction of these proteins in IP is due to the loss of a direct or indirect interaction involving Sgcg.

We used immunofluorescence co-localization in human rhabdomyosarcoma cells (RH30) as a secondary screen to interrogate potential interactions of the SC with PP1 $\beta$ and the PP1 $\beta$-interacting proteins in a cell type that lacks high-level muscle protein organization. The rationale was that signal proximity in the absence of large assemblages of muscle proteins would be consistent with an association requiring only a few proteins. We used cDNAs encoding tagged NKCC1, PP1 $\beta$, MYPT2, and tensin2 co-expressed with either untagged Sgcg or Sgcg-Flag, with and without co-expression of Sgcb-Flag and Sgcd-Flag; together these three sarcoglycans form the SC core [51, 64]. We also interrogated protein localizations with antibodies, as available, including two different, specific Sgcg antibodies (Additional files 7, 8, and 9; Figs. S4, S5, and S6).

\section{NKCC1 is an Sgcg interactor}

The novel candidate interactor with the strongest colocalization with co-expressed Sgcg in RH30 cells was NKCC1 (Fig. 4). RH30 cells express reduced levels of skeletal muscle proteins, supporting assessments of direct or indirect protein-protein interactions independent of the presence of an organized contractile apparatus [78]. Positive co-localizations of NKCC1 with Flag-tagged SC proteins were confirmed using Sgcg antibody (Fig. 4A, arrows, vs. B) and anti-Flag (Fig. 4C). Most co-localizations were seen in punctate structures suggestive of intracellular vesicles. To test whether these overlaps were due solely to co-existence of these membrane proteins in the same lipid bilayer, we briefly extracted RH30 cells expressing both proteins with detergent before fixation and staining. Under these conditions, the cytosolic control HA-CFP diffused away (Fig. 4D), but the signals from NKCC1 and sarcoglycans remained, as did the pronounced signal overlaps (Fig. 4C, magenta).

Less pronounced signal overlaps in transfected RH30 cells were observed for the sarcoglycans and a fusion protein of HA-CFP with a truncated NKCC1 protein containing the cytosolic $\mathrm{N}$-terminus directly fused with the cytosolic C-terminus (NKCC1-cyto); this NKCC1 truncation lacks the 12 transmembrane domains and extracellular sequences [56] (Fig. 5). Unlike fulllength NKCC1 (Fig. 4A), this NKCC1-cyto protein was 


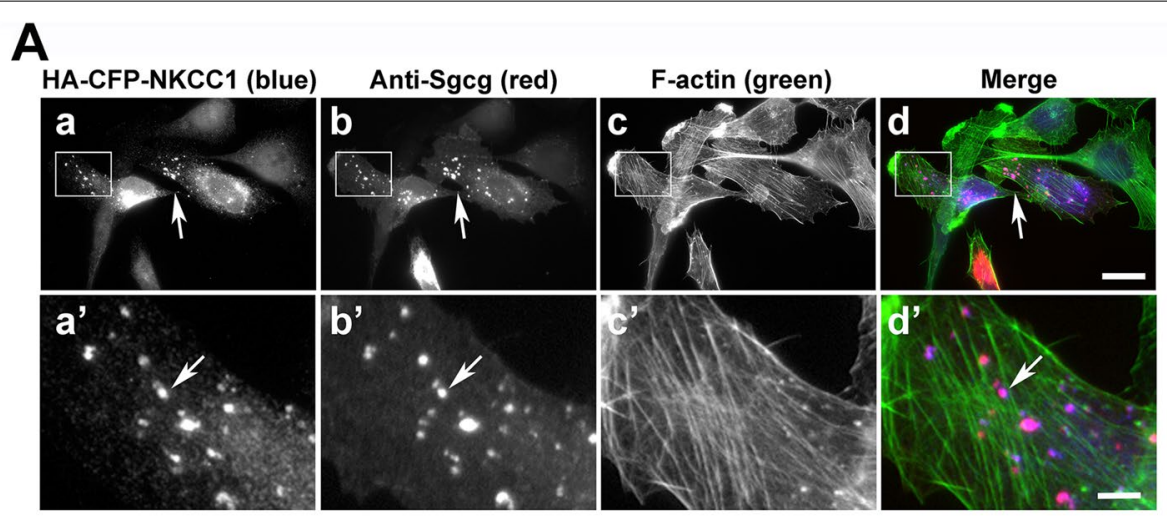

B

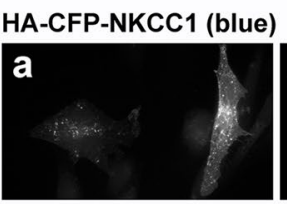

Rb lgG (red)
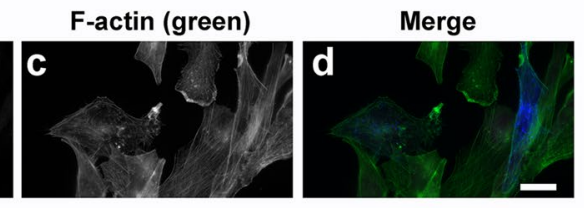

C

Brief Detergent Extraction before Fixation
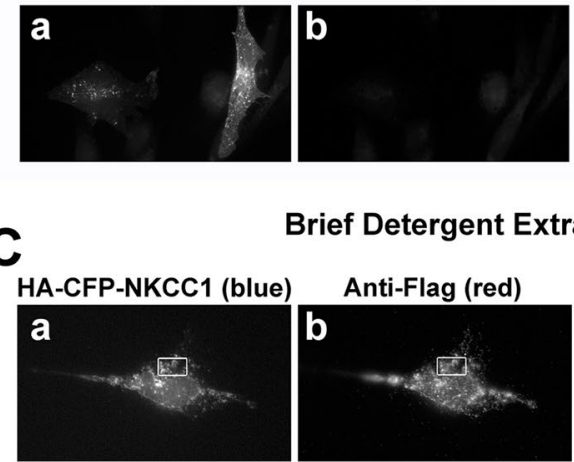

Anti-Flag (red)
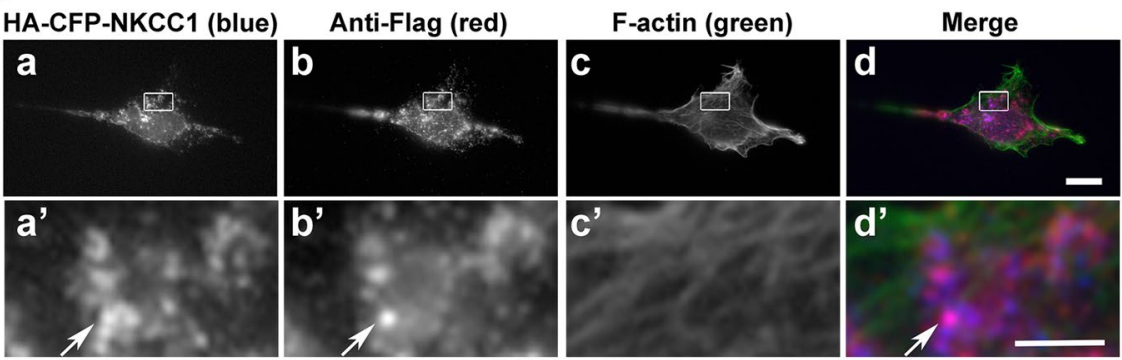

D
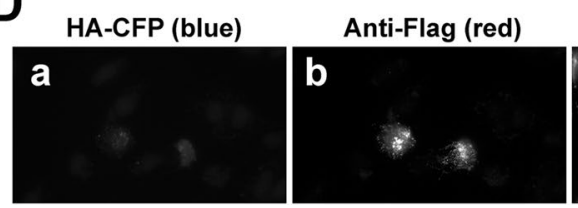

\section{F-actin (green)}
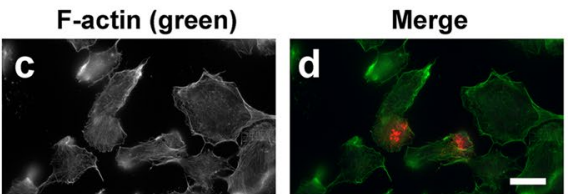

Fig. 4 NKCC1 co-localizes with the sarcoglycan complex in large, detergent-stable vesicular structures. A, B HA-CFP-tagged NKCC1 (panels a, a', blue in merges) co-localizes in punctae visualized with both $\mathbf{A}$ our affinity-purified anti-Sgcg and $\mathbf{B}$ anti-Sgcg from Proteintech Group (PTG) (panels b, b', red in merges) after expression in RH30 rhabdomyosarcoma cells with Flag-tagged Sgcg, Sgcd, and Sgcb. Enlargements shown (a'-d') of the boxed areas in panels a-d. C Specificity control with nonimmune rabbit (Rb) IgG substituted for anti-Sgcg. D Signal overlaps of HA-CFP-NKCC1 (panels a, a', blue in merges) and the sarcoglycan complex (panels a, a', red in merges) persist even when cells are briefly extracted with $0.1 \%$ Triton X-100 before fixation. E The HA-CFP tag alone (panel a, blue in merge) does not persist in pre-extracted RH30 cells co-transfected with Flag-tagged Sgcg, Sgcd, and Sgcb (panel a, red in merge). A-E Phalloidin-stained actin filaments delineate cell boundaries (panels c, c', green in merges). Bars (d), $20 \mu \mathrm{m}$; bars (d'), $5 \mu \mathrm{m}$. Overlapping HA and Flag signals in magenta (arrows)

predominantly cytosolic (Fig. 5A); the cytosolic signal was intensified in the vicinity of sarcoglycan punctae (Fig. 5A, arrows). More of the NKCC1-cyto protein was located in the cytosol than was observed for the more nuclear HA-CFP control protein (Fig. 5B). Both the NKCC1-cyto (Fig. 5C) and the HA-CFP (Fig. 5D) proteins diffused away from the sarcoglycan punctae in cells pre-extracted with detergent, suggesting that any association between the SC and the NKCC1 cytoplasmic domains must be of low avidity.

By contrast, we observed no co-localization of sarcoglycans with tagged tensin 2 at focal adhesions (Additional file 10, Fig. S7) and limited signal overlap with MYPT2 (Additional file 11, Fig. S8) or PP1 $\beta$ (Additional file 12, Fig. S9). MYPT2 localized primarily with phalloidin-stained actin filaments (Additional file 11, Fig. S8), 


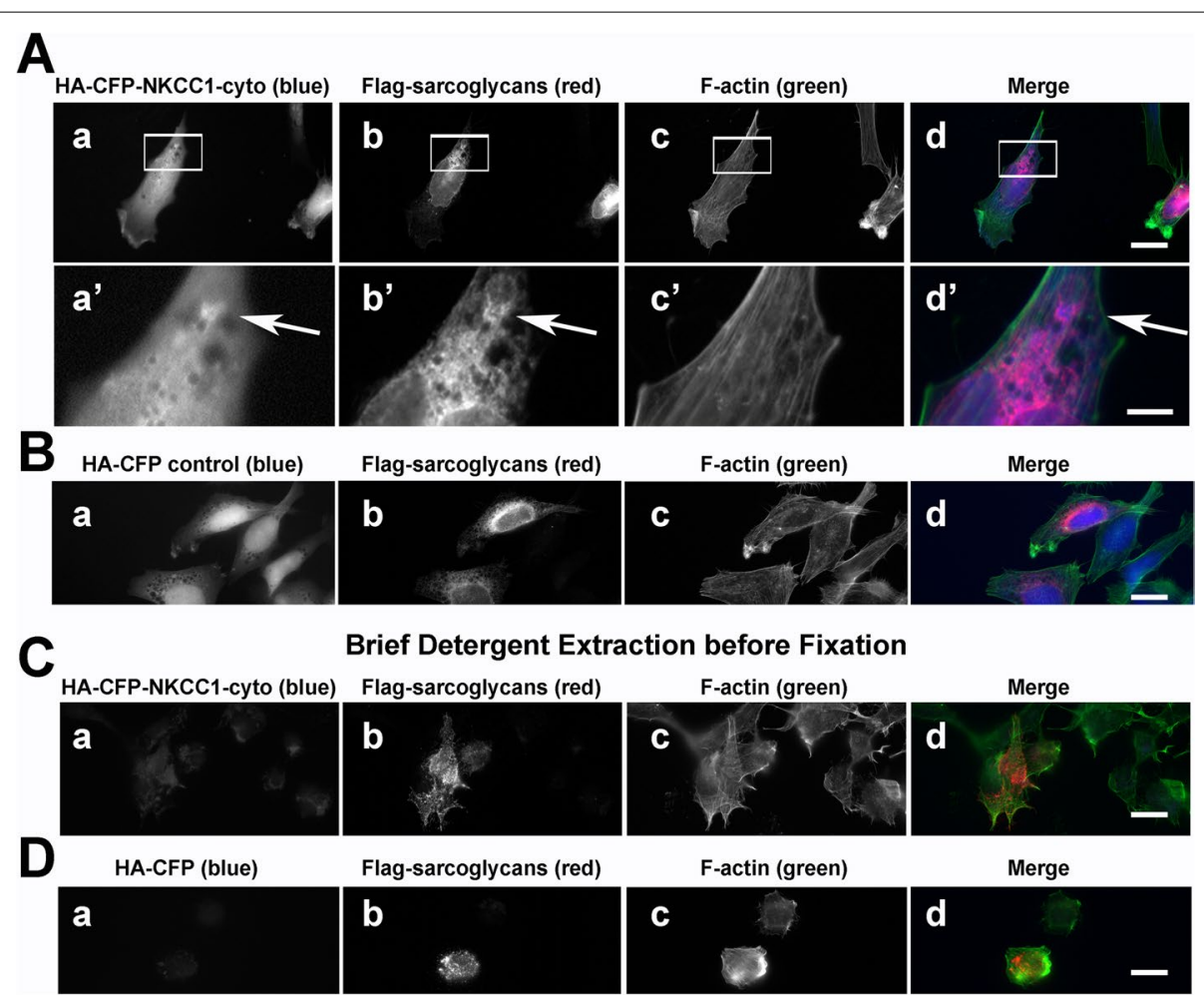

Fig. 5 HA-CFP-NKCC1-cytoplasmic domains appear to be loosely associated with Flag-tagged SC proteins. RH30 cells were co-transfected in parallel with Flag-tagged sarcoglycans and stained as in Fig. 4. A HA-CFP-NKCC1-cytoplasmic domains and B HA-CFP (negative control; panels a, $a^{\prime}$; blue in merges) were visualized with Flag-tagged sarcoglycans (panels b, b'; red in merges) and F-actin (panels $c, C^{\prime}$; green in merges). After pre-extraction with $0.1 \%$ Triton X-100, C signal from residual HA-CFP-NKCC1-cytoplasmic domain was indistinguishable from D residual HA-CFP. Overlapping HA and Flag signals in magenta (arrows). Bars (d), $20 \mu \mathrm{m}$; bar (d'), $5 \mu \mathrm{m}$

suggestive of an interaction with myosin II in stress fibers [79]. In some cells, MYPT2 overlapped with brightly stained juxtanuclear Sgcg punctae, but no associations with large Sgcg-containing punctae in the cell periphery were observed (Additional file 11, Fig. S8). Similarly, signals from co-expressed Sgcg and PP1 $\beta$ tagged at either end with EGFP were not clearly different from the EGFP control (Additional file 12, Fig. S9), even under hypoosmotic conditions that induce translocation of PP1 $\alpha$ from the nucleus to the cytoplasm [80]. These negative results do not eliminate the possibility of an interaction with Sgcg, but suggest that any binding to PP1 $\beta$ or MYPT2 requires either post-translational modifications or muscle-specific interaction partners.

\section{Sgcg interacts with NKCC1 cytoplasmic domains}

To further examine the interactions of the SC with NKCC1 and the NKCC1-cyto domains, we co-overexpressed the tagged proteins in $\mathrm{RH}-30$ cells and used the tags for co-IP, supernatant-depletion assays, and PLA (Fig. 6). Initial co-IP experiments using brief, low-stringency washes revealed that more HA-CFP-NKCC1 and NKCC1-cyto protein than HA-CFP negative control co-sedimented with Flag-tagged sarcoglycans (Sgcb, Sgcd, Sgcg) (Fig. 6A). However, signal was lost if cell lysates were subjected to freeze-thaw or if the IP pellets were washed stringently, suggesting that any interaction was of low avidity in vitro. We therefore pursued supernatant-depletion assays for better quantification of lowavidity interactions [61]. In these assays, the amount of the Unbound partner protein in the supernatant is monitored before and after sedimentation of the bound protein to quantify the initial binding equilibrium [61]. The Unbound-to-Input ratio reveals the amount of target protein lost with the bound pellet, without a requirement that the interaction withstands the dilutions and time required for rinsing. Ratios less than 1 are consistent with a binding interaction. We consistently found Unbound/ Input ratios less than one for Sgcg-Flag sedimented by full-length HA-CFP-NKCC1 or HA-CFP-NKCC1-cyto, as compared to the HA-CFP control protein, although only the result with HA-CFP-NKCC1-cyto was statistically significant due to the variability of the control (Fig. 6B, C). The only observed Flag-associated signal was from Sgcg-Flag, possibly due to the presence of endogenous Sgcb and Sgcd in these cells (https://www.proteinatl 

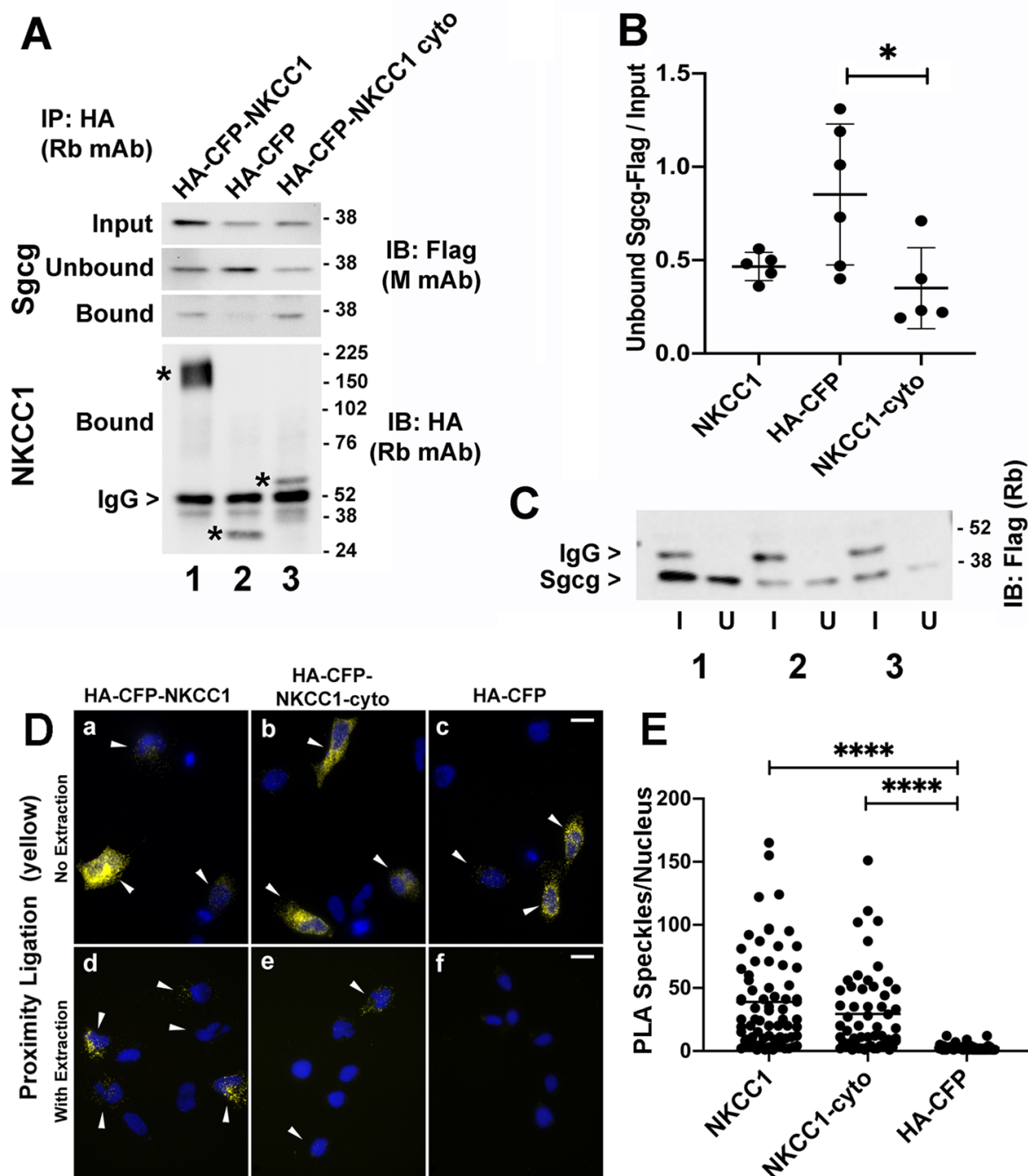

HA-CFP-

HA-CFP
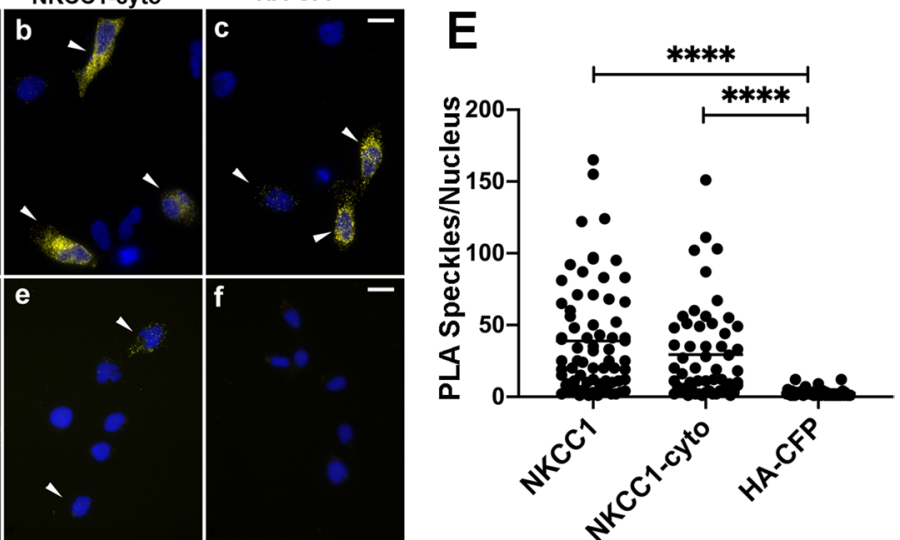

Fig. 6 Flag-tagged Sgcg directly or indirectly associates with HA-CFP-tagged NKCC1 proteins. A Immunoblot of the most successful co-IP, showing more Sgcg-Flag sedimenting with the NKCC1 and NKCC1-cyto (bound), compared to the HA-CFP negative control; corresponding decreases were observed in supernatants after sedimentation (Unbound). Sgcg-Flag amounts in cell lysates also are shown (Input). Flag-tagged Sgcg, Sgcb, and Sgcd proteins were co-expressed with either HA-CFP-NKCC1 (lane 1), HA-CFP (lane 2), or HA-CFP-NKCC1 cytoplasmic domains (NKCC1-Cyto, lane 3) in RH-30 cells and immunoprecipitated with anti-HA. Bound NKCC1 proteins were visualized with anti-HA (asterisks); bead-bound rabbit anti-HA heavy chain (IgG). B Densitometric quantification of Unbound/Input ratios of Sgcg-Flag from supernatant-depletion assays, such as those shown in $\mathbf{C}$, and analysis by one-way ANOVA with Tukey's multiple comparisons test. $P$ values for the mean differences between HA-CFP control and HA-CFP-NKCC1 and HA-CFP-NKCC1-Cyto were 0.12 and 0.02 , respectively $\left.{ }^{*} P<0.05\right)$. Central bars, means; error bars, S.D. C Representative immunoblot for supernatant-depletion assays in $\mathbf{B}$, showing decreases in band intensity in Unbound lanes (U), compared to Inputs (I). IP used rabbit monoclonal anti-HA (HA); Sgcg-Flag was visualized with rabbit anti-Flag (Flag); HA-CFP-tagged NKCC1 (lane 1), HA-CFP alone (lane 2), or HA-CFP-NKCC1 cytoplasmic domains (lane 3). D, E Representative images and quantification of PLA for the SC interaction with HA-CFP-NKCC1, HA-CFP-NKCC1-cyto, or the HA-CFP control in RH30 cells co-transfected with Flag-tagged Sgcg, Sgcb, and Sgcd. D In cells fixed without detergent pre-treatment to remove free cytosolic proteins (Figs. 4D and 5D), PLA speckles indicating proximity of anti-Sgcg and anti-HA were observed with all three HA-CFP constructs (Da-C). A brief detergent pre-extraction revealed persisting proximity of the SC complex with NKCC1 and NKCC1-cyto, but not with the HA-CFP tag alone (Dd-f, E). PLA speckles are pseudocolored yellow; DAPI-labeled nuclei in blue. Size bars, $20 \mu \mathrm{m}$. E Graph of PLA speckles per nucleus for pre-extracted cells. Cells with no PLA speckles were assumed to be untransfected and were not included. Horizontal bars, means from results combined from 2 experiments with the following total numbers of counted cells: NKCC1 (70), NKCC1-Cyto (56), HA-CFP (65). Transfection efficiencies varied from $36 \%$ to $65 \%$, with higher values for HA-CFP, despite a lower percentage of PLA speckles. ${ }^{* * * *} P<0.0001 ; P$ for NKCC1 versus NKCC1-cyto was 0.6140 (ns), from a one-way ANOVA with a Kruskal-Wallis multiple comparisons test 

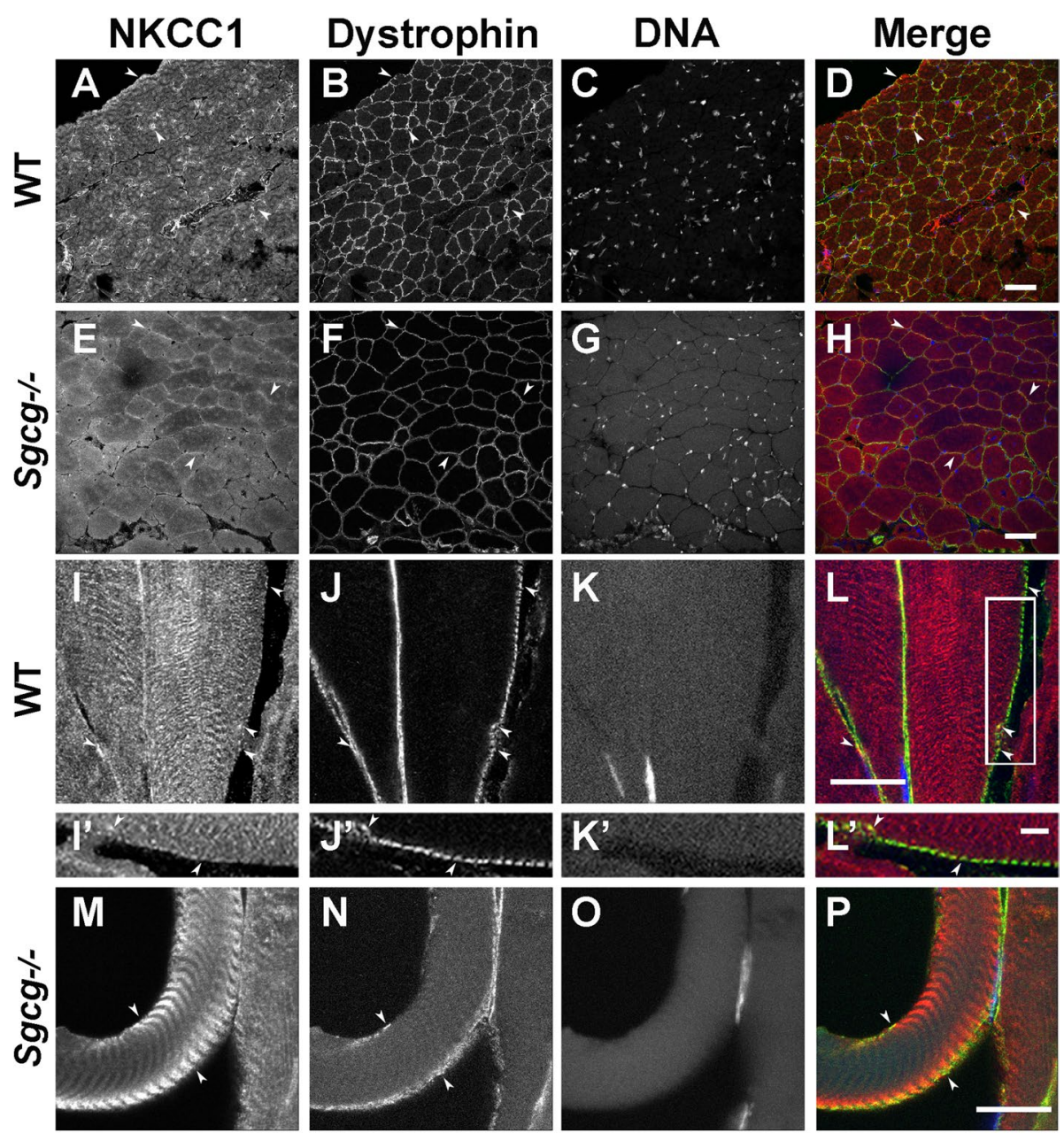

\section{Secondaries only}
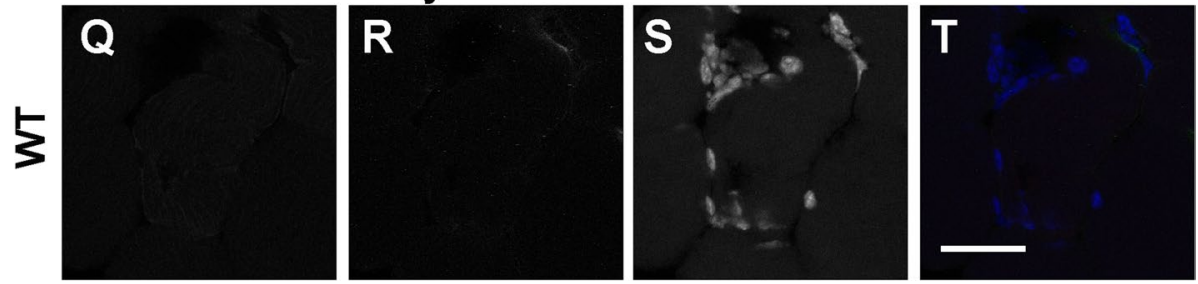

Fig. 7 Confocal microscopy of NKCC1 and dystrophin in EDL muscles from WT and $\mathrm{Sgcg}^{-/-}$mice. A-H Cross sections show overlapping signals at the sarcolemma (arrowheads; yellow in merges), as well as internal myofibrillar NKCC1 staining in both genotypes. I-P In longitudinal sections, the internal staining suggests an association with T-tubules, which end at dystrophin-associated costameres in the sarcolemma [8, 9, 82]. $\mathbf{I}^{\prime}-\mathbf{L}^{\prime}$ Enlargements of the area boxed in L. Q-T Sections stained with only secondary antibodies. Bars are A-H $50 \mu \mathrm{m} ; \mathbf{I}-\mathbf{T} 20 \mu \mathrm{m} ; \mathbf{I}^{\prime}-\mathbf{L}^{\prime} 5 \mu \mathrm{m}$

as.org). By contrast, supernatant-depletion assays with co-expressed Flag-tagged sarcoglycans and PP1 $\beta$ tagged with EGFP at either end resulted in no ratios of Unbound to Input less than 1 in co-IP experiments that used either anti-Flag or anti-EGFP. PLA of transfected cells further supported the existence of a direct or indirect interaction between the SC and NKCC1 (Fig. 6D, E). In unextracted co-transfected cells, all HA-CFP tagged constructs showed proximity with the SC (Fig. 6D, top row). After pre-extraction with low levels of detergent to eliminate free cytosolic proteins, significantly more PLA signal was observed for the SC with NKCC1 and NKCC1-cyto than with the HA-CFP negative control (Fig. 6D, E). These results support the existence of a low-avidity association between exogenously expressed sarcoglycans and the NKCC1 cytoplasmic domains. 


\section{NKCC1 co-localizes with the DGC and affects} Sgcg-dependent ERK activation in muscle

To see if NKCC1 localizes in the vicinity of the SC and thus might associate in vivo, we localized NKCC1 in murine EDL muscles (Fig. 7). NKCC1 was known to be in skeletal muscle fibers [81], but its localization with respect to dystrophin at costameres in muscle fibers $[8,9]$ was unexplored. In immunostained muscle cryosections, the anti-NKCC1 signal exhibits the same distribution as that of sarcolemmal dystrophin in both the presence of Sgcg in control muscle (Fig. 7A, B, I, J) and in its absence in muscles from $\mathrm{Sgcg}^{-/-}$mice (Fig. 7E, F, M, N). Additional NKCC1 signal is associated with internal striations (Fig. 7I, M), consistent with localization at or near the T-tubule network that synchronizes calcium release within the muscle, and appears to be concentrated in the vicinity of T-tubule mouths at costameres $[82,83]$. The similar signal distributions in the presence and absence of the SC indicate that $\mathrm{NKCC1}$ does not require Sgcg for expression and transport to the sarcolemma. These results place $\mathrm{NKCC1}$ into proximity with the DGC in vivo.

We also explored whether NKCC1 co-transporter activity affects SC-mediated ERK signaling [18, 24]. NKCC1 co-transporter activity increases $\mathrm{K}^{+}$-induced calcium transients in muscle by facilitating the influx of the chloride counter ion [84]. We found that NKCC1 activity also alters P-ERK responses. The NKCC1 co-transport inhibitor bumetanide had no significant effect on the specific forces of EDL muscle contraction or the relative loss of force following a series of eccentric contractions (Fig. 8A, B). However, increased P-ERK was evident in resting WT muscle incubated in bumetanide (Fig. $8 \mathrm{C}$ ). After eccentric contractions (ECC), bumetanide inhibited ERK phosphorylation in $\mathrm{Sgcg}^{-/-}$muscles, but not in WT muscle (Fig. 8D, E). Sgcg-dependent changes in total glycosylated NKCC1 and in NKCC1 phosphorylation, a measure of NKCC1 activation [66, 85, 86], might occur during ECC strain, but the differences were not statistically significant (Additional file 13; Fig. S10). These results support a role for NKCC1 in Sgcg-dependent ERK activation in response to ECC [18], without a Sgcgdependent alteration of NKCC1 phosphorylation.

\section{New candidate Sgcg interactors link the SC with survival signaling pathways in silico}

We also used in vitro assays to evaluate the directness of the interaction between Sgcg and archvillin, a membrane-cytoskeleton linker protein that is a scaffold for ERK1/2 signaling $[27,30]$. This interaction was originally identified with yeast two-hybrid assays and supported by co-IP [26]. Because initial co-IPs suggested a low-avidity interaction similar to that observed for Sgcg and NKCC1 proteins, we carried out supernatant-depletion assays with purified, recombinant Sgcg $\mathrm{N}$-terminus (Sgcg 1-35-EGFP, WT) and the conserved archvillin/ supervillin C-terminus (Fig. 9). Soluble EGFP and Sgcg 1-35-EGFP proteins were purified and used with purified GST-tagged archvillin C-terminus (Fig. 9A, B) or GST alone (Fig. 9C, D) in GST-pulldown assays. We observed a statistically significant direct interaction between the archvillin C-terminus and WT Sgcg (Fig. 9A, B). In addition, Sgcg 1-35-EGFP with a mutation in Tyr-6 (Y6A), the residue implicated in P-ERK signaling in muscle [24], exhibited a mean Unbound/ Input ratio that was intermediate between that observed with Sgcg WT and the EGFP control, but was not statistically significant from either value. No differences among the proteins were observed with the GST control beads (Fig. 9C, D). These results support the directness of the previously described Sgcg interaction with archvillin [26] and suggest that Sgcg-Y6 may be involved in the Sgcg-archvillin interaction.

The Sgcg-archvillin direct interaction and the new candidate direct or indirect interactors reported in Fig. 3 were then analyzed using IPA software to graphically integrate the new associations described here with published data [87] (Fig. 10). Most candidate proteins fell into one of two pathways: (1) the dystrophin- and sarcoglycan-associated DGC or (2) an interconnected signaling pathway containing p53 (gene name TP53), estrogen receptor $\beta$ (ESR2), and the ubiquitin E3 ligase TRIM25. Eleven of the 13 top candidate interactors (Figs. 3 and 10, blue), including NKCC1 (gene Slc12a2, green), archvillin (Svil, yellow), and 3 of the 5 other candidate interactors (Fig. 10, gray) identified in Sgcg IPs (Fig. 3), were assigned by the program as components of this survival signaling pathway or the sarcolemmal network. With the addition of the Sgcg-archvillin interaction [26] (Fig. 9) and the new Sgcg candidate interactors (Fig. 3), the software readily merged the sarcolemmal and signaling pathways into a single larger network (Fig. 10). We then manually added additional links to show the published scaffolding of ERK and the ERK-activating kinase MEK2 by $\beta$-dystroglycan (gene $D A G$ ) [88], the scaffolding of ERK and the MEK1/2-activating kinase B-Raf (gene $B R A F$ ) by the smooth muscle isoform of archvillin (gene SVIL, Fig. 10, yellow) [30], and the interaction demonstrated here between Sgcg (Fig. 10, pink) and NKCC1 (gene SLC12A2) (Figs. 4, 5, and 6). The merger of these interactors into a single network in silico is consistent with a role for sarcoglycan interactors in ERK regulation and survival signaling in muscle. 

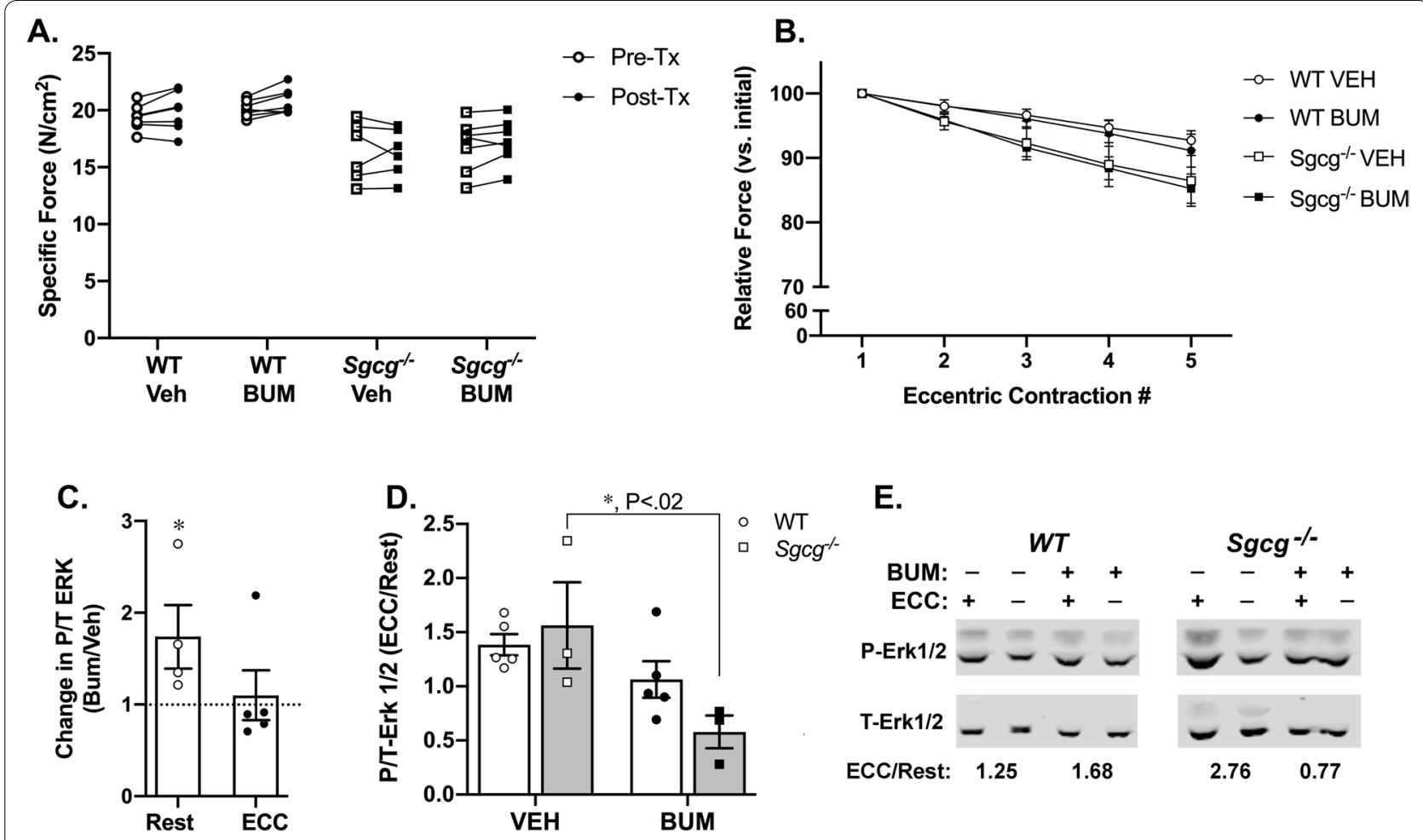

Fig. 8 Effects of NKCC1 inhibition on muscle force generation and ERK phosphorylation. A Isometric tetanic force generation of EDL muscles is not altered by bumetanide (BUM) incubation, as compared with vehicle (VEH) control. Raw values showing forces in Ringer's solution as pre-treatment (Pre-Tx), and the forces following 20-30-min incubation in $50 \mu \mathrm{M}$ bumetanide or vehicle (ethanol) as post-treatment (Post-Tx). B Decrement of force following eccentric contractions (ECC) is not affected by bumetanide incubation. Forces are relative to the initial force in the first ECC. Means shown; error bars SEM. C levels of phosphorylated ERK are elevated in resting EDL muscles from wild-type mice, but the P-ERK response to ECC is unaffected. (*,$P<0.05$, paired t-test between bumetanide and vehicle incubation for muscles from the same mouse). $D$ Bumetanide incubation inhibits ECC-induced increases in P/T-ERK in the absence of Sgcg. $N=3-6$ muscles per condition. *Significant difference between vehicle and bumetanide within strain $(P=0.0196)$, by 2-way ANOVA followed by Sidak's multiple comparisons test. C, D Central lines, means; error bars, SEM. E Representative immunoblots. The signals from phosphorylated (P) to total $(T)$ ERK signal were determined for each condition, and then the ratio of the $\mathrm{P} / \mathrm{T}$ ERK signals after ECC were divided by the corresponding ratio at rest for each condition (ECC/Rest)

\section{Discussion}

Using a proteomics approach optimized for recovery of muscle membrane proteins, we identified novel candidate direct or indirect interactors for Sgcg, and by extension, the $\mathrm{SC}$. Our screen identified all subunits of the SC, plus 16 other proteins that specifically co-IP with a high-avidity Sgcg antibody from muscles that contain the SC but are reduced in muscles from $\mathrm{Sgcg}^{-/-}$mice, which lack these proteins (Fig. 3). Differences in our approach versus those in previous studies include prior enrichment for membranes and the analysis of cytoskeleton-associated, detergent-resistant membranes using an IP buffer with SDS. The use of $\mathrm{Sgcg}^{-/-}$muscles as a specificity control eliminates proteins present in the co-IPs due to low-avidity antibody cross-reactivities not recognizable by immunoblots or IF (Additional files 3 and 4; Tables S2 and S3). It is especially noteworthy that most of the identified top and other candidate interactors in Fig. 3 were readily grouped in silico into a single signaling pathway that links ERK regulation with survival signaling (Fig. 10).

NKCC1 emerged as a potential interactor with Sgcg in a secondary screen, which consisted of co-localizing exogenously expressed SC proteins and candidate interactors (Fig. 4, Additional files 10, 11, and 12, Figs. S7, S8, and S9). Immunofluorescence co-localization (Fig. 5), co-IP (Fig. 6A), supernatant-depletion assays (Fig. 6B, C), and PLA (Fig. 6D, E) were consistent with a low-avidity direct or indirect interaction between the SC and the NKCC1 cytoplasmic domains that may be comparable in magnitude to the direct, low-avidity binding of the $\mathrm{Sgcg}$ $\mathrm{N}$-terminus to the archvillin C-terminus (Fig. 9). We also show that NKCC1 overlaps with dystrophin at skeletal muscle costameres and with internal muscle striations (Fig. 7), sites that are likely T-tubules involved in intracellular $\mathrm{Ca}^{++}$regulation [82, 89]. Physiological analyses with the NKCC1 inhibitor bumetanide also suggest a role for $\mathrm{Na}^{+} \mathrm{K}^{+} 2 \mathrm{Cl}^{-}$co-transport during SC-mediated ERK 

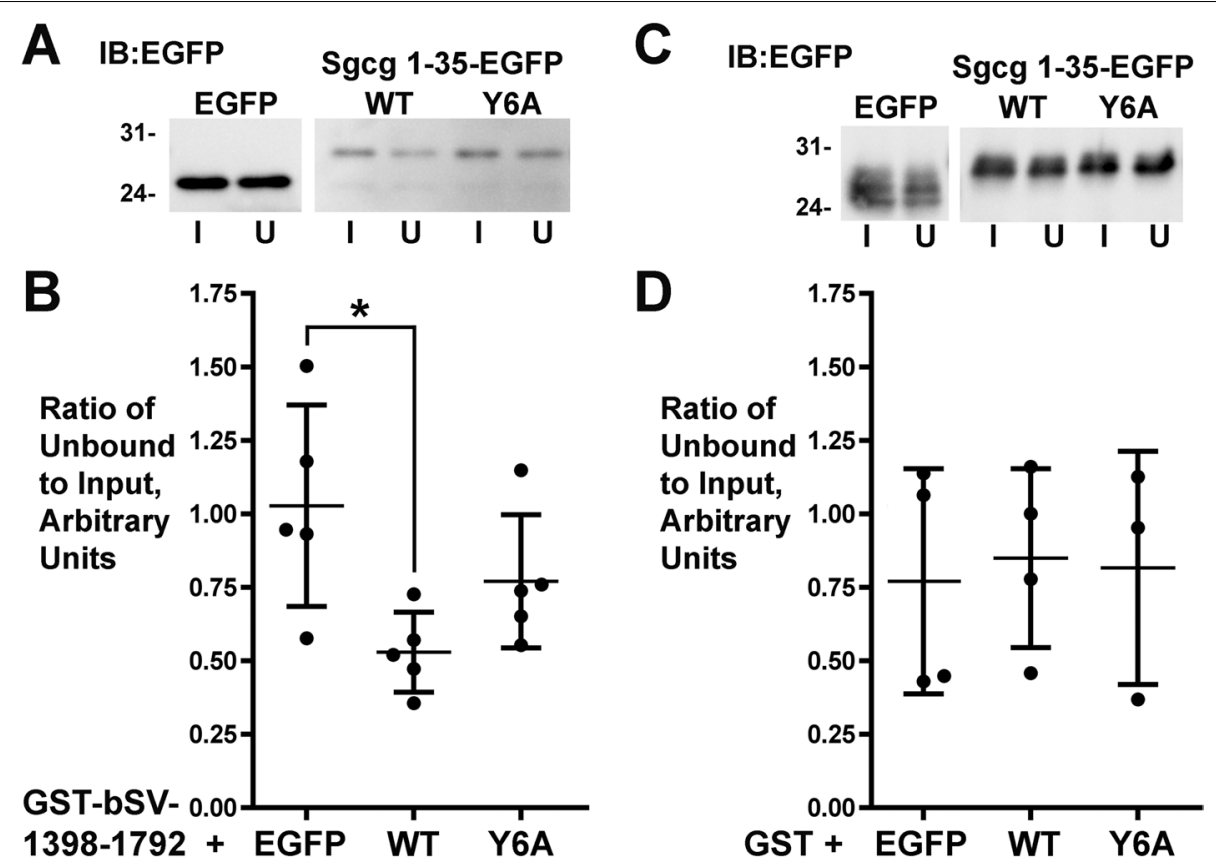

Fig. 9 Direct binding of recombinant Sgcg N-terminus to the conserved archvillin/supervillin C-terminus. Supernatant depletion assays showed a significant loss of recombinant Sgcg-1-35-EGFP, but not of recombinant EGFP, after pull down with A, B GST-tagged bovine supervillin (bSV) residues 1398-1792 or C, D GST alone. Mutation of tyrosine-6 in Sgcg to alanine (Y6A) may affect this interaction. A, C Representative immunoblots of Input (I) and Unbound (U) fractions. B, D Densitometry quantification of replicate experiments. Statistical analyses employed one-way ANOVA, with a Tukey-Cramer multiple comparisons post-test. $N=3-5$, as shown; ${ }^{*}<0.05$. Central lines, means; error bars, S.D.

signaling in skeletal muscle (Fig. 8). The NKCC1 localization in proximity to T-tubules and the apparent role of NKCC1 co-transport in sarcoglycan-regulated signaling after ECC strain support a direct or indirect interaction of NKCC1 with the SC.

The high-avidity association observed between fulllength NKCC1 and Sgcg during co-IP in the SDS-containing RIPA1 buffer (Fig. 3) and after pre-extraction with detergent in IF (Fig. 4) suggests that this association involves more than the NKCC1 cytoplasmic domains used in our in vitro assays (Fig. 6). Interactions observed in supernatant-depletion assays or PLA, such as those of Sgcg with the NKCCI cytoplasmic domains (Fig. 6) and with archvillin (Fig. 9), may be of relatively low avidity. A low-avidity interaction between Sgcg and archvillin is consistent with its identification in yeast two-hybrid assays [26], which also detect weak interactions [90]. Low-avidity interactions are often cumulative, regulated by post-translational modifications, and known to play important roles in signaling networks [91, 92]. Other interactions involving the $\mathrm{NKCC} 1$ transmembrane or extracellular domains are likely required for a tight association with Sgcg in vivo, either through direct binding or by indirect associations involving other proteins in this emerging signaling network. Transmembrane domains, by themselves, can enhance the avidity of an association $\sim 100$ fold by orienting and concentrating both partners at the membrane surface [93]. Multiple connection(s) between NKCC1 and the DGC also are suggested by the SC-independent localization of NKCC1 in $\mathrm{Sgcg}^{-/-}$muscles (Fig. 7). Thus, the DGC-associated signaling network may consist of many post-transcriptionally regulated interactions with variable and regulatable avidities.

Although the results from the secondary screens involving PP1 $\beta$, MYPT2, and tensin did not support an interaction with the $\mathrm{SC}$, these results do not eliminate the possibility of indirect or conditional associations. For instance, PP $1 \beta$ may link indirectly to Sgcg through binding to NKCC1 [66]. Furthermore, assays with PP1 $\beta$ in cell lysates are challenged by the presence of the $\sim 200$ known PP1 $\beta$-binding proteins that could act as endogenous competitive inhibitors [94]. Regulation of PP1 $\beta$ localization or function at costameres by Sgcg is still possible, given the cross-talk between the SC and P-ERK signaling $[18,20,24]$.

Our novel approach has expanded the list of candidate SC-interacting proteins and thus complement results from previous studies $[95,96]$. Most of our candidate interactors were obtained from a cytoskeleton-associated membrane fraction that has been typically not interrogated. Also, the inclusion of SDS in the RIPA1 buffer may have disrupted many previously reported interactions. 


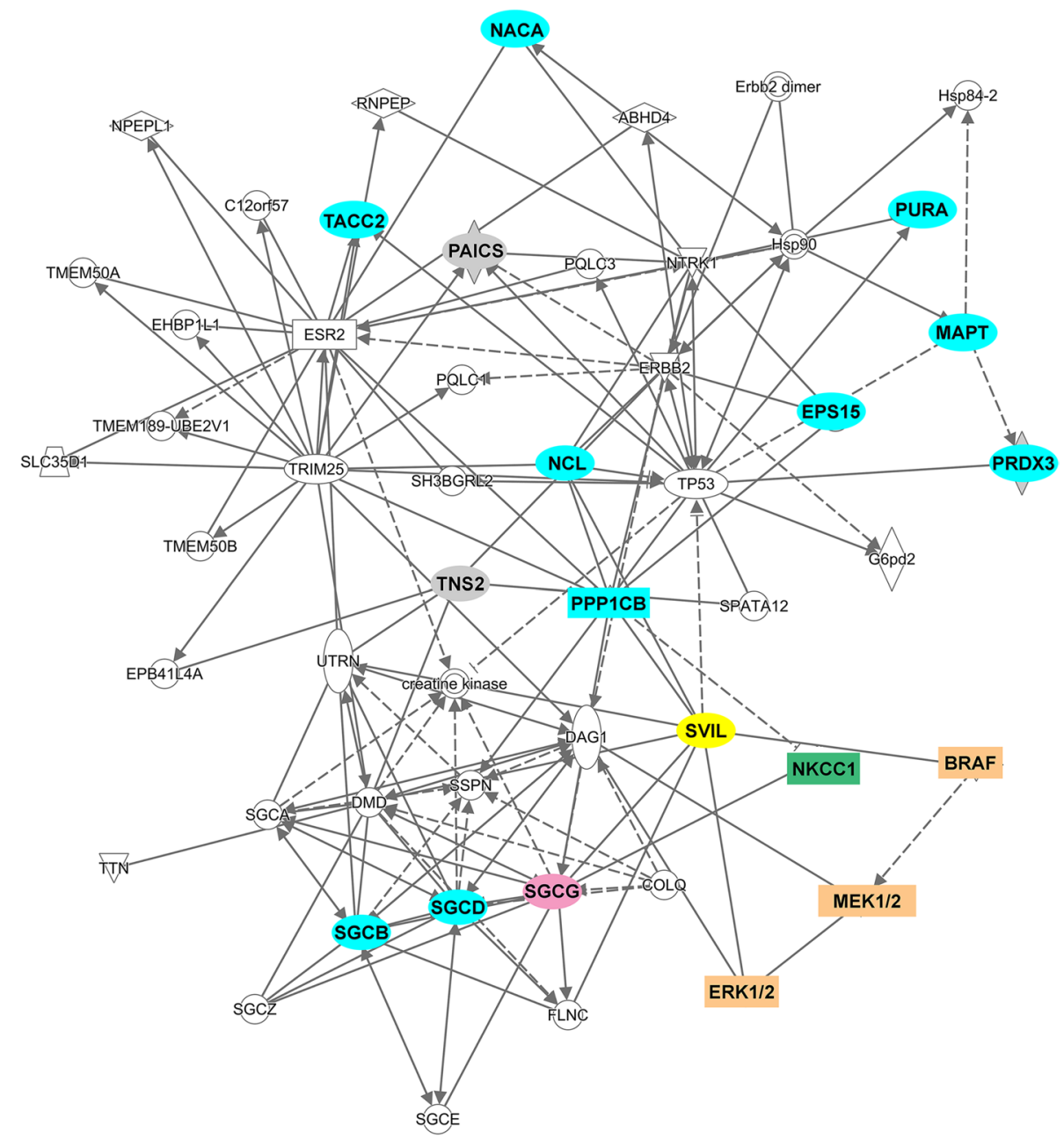

Fig. 10 Pathway analysis suggests cross-talk between interconnected survival signaling pathways and sarcolemmal sarcoglycans, dystrophin, and archvillin. The gene names of proteins co-immunoprecipitating with or binding to Sgcg as shown here were arranged in the default Organic View using IPA software. Eleven of 13 Top Candidate Sgcg interactors, as well as 3 of 5 Other Candidate interactors (Fig. 3), were assigned to either the TP53/ESR2/TRIM25-associated signaling network or to the sarcoglycan, dystrophin, dystroglycan-associated sarcolemmal network. Colors highlight SGCG (magenta), SVIL (yellow), NKCC1/SLC12A2 (green), additional Top Candidate Interactors (blue) and Other Candidate Interactors (gray). Additional genes (white) and direct (solid lines) and indirect (dashed lines) interactions were added by the IPA software. Interactions involving the B-Raf, MEK1/2, and ERK1/2 signaling cascade (orange) with $\beta$-dystroglycan/DAG [88] and SVIL [30] were added manually

For instance, $\beta$-dystroglycan is a well-accepted interactor of the SC $[5,97]$ that is present in our RIPA1 lysate (Fig. 2E), but did not appear as a specific interactor in our co-IPs (Fig. 3). The accepted $\beta$-dystroglycan-SC interaction emerged from co-IPs with nonionic detergent extracts of membranes not tightly bound to the cytoskeleton $[95,96]$. The procedural differences may explain the absence of $\beta$-dystroglycan and other published SC-associated proteins from our list of candidate interactors.

A role for NKCC1 in skeletal muscle mechanotransduction is consistent with prior studies. NKCC1 has long been appreciated as necessary for muscle volume control, which is regulated by P-ERK under osmotic, but not hyperosmotic, conditions [98]. NKCC1 is implicated in myoblast differentiation and exerciseinduced hypertrophy of skeletal muscle [84], as well as in phenylephrine-mediated rhythmic contractions of smooth muscle [99]. NKCC1 activity also promotes transient decreases in muscle force in mouse models of hypokalemic periodic paralysis $[100,101]$. The mechanism of action is thought to involve increased transient accumulations of intracellular calcium ions that are enhanced by NKCC1-dependent increases in the chloride counter-ion concentration subjacent to the sarcolemma [99, 102, 103]. ECC promotes $\mathrm{Ca}^{++}$entry across the sarcolemma [104], and increased calcium influx 
induced by a number of genetic mechanisms is sufficient to induce muscular dystrophy $[105,106]$. Thus, NKCC1 function at the SC can be plausibly linked to molecular events leading to long-term muscle damage.

Our physiological analyses suggest that NKCC1 transporter activity reduces ERK activation in resting wildtype muscle and promotes a SC-dependent increase in P-ERK after ECC. ERK1/2 activation protects myofibers from damage and contributes to cardiac hypertrophy and protein expression of dystrophin and utrophin in skeletal muscle [29, 107, 108]. NKCC1 transporter activity thus may be antagonistic to ERK activation in resting muscle and contributory to stimulus-induced ERK activation after ECC. In fact, prior studies have positioned NKCC1 activity both upstream and downstream of ERK activation. Inhibition of ERK signaling in tracheal epithelial cells reduced bumetanide-sensitive ${ }^{36} \mathrm{Cl}^{-}$uptake, a measure of NKCC1 activity [109]. However, NKCC1 inhibition or depletion attenuated P/T ERK phosphorylation in fibroblasts [110, 111], astrocytes [112], HeLa cells [113], and injured rat brains [114]. Epidermal growth factor stimulation of corneal epithelial cells promoted co-IP of NKCC1 and P-ERK1/2 [115], suggesting stimulus-mediated association. Up-regulation of P-ERK1/2 also is consistent with the anti-apoptotic activity of NKCC1 in cancer cells [116]. Thus, NKCC1 provides a route through which the SC could coordinate $\mathrm{Ca}^{++}$and P-ERK signaling, with the differences between resting and stimulated muscle due to regulation of NKCC1 scaffolding of PP1 $\beta$ and signaling kinases [66]. Muscle strain also may affect coordination or competition between the NKCC1 and archvillin/kinase/PP1 $\beta$ scaffolds in binding to the SC.

The regions of signal overlap between NKCC1 and dystrophin in muscle imply proximity to costameres, the probable site of SC signaling. Costameres are structured lipid microdomains rich in caveolin-3, cholesterol, detergent-insoluble lipids, and cytoskeletal proteins and are a type of "lipid raft" [117]. Cholesterol-rich lipid rafts regulate many ion channels through altered conformations or associations with other proteins $[118,119]$. Loss of caveolin-3 results in loss of costameric Sgca and enhanced ERK signaling [117]. NKCC1 co-fractionates from rat brain with lipid raft-associated proteins, and NKCC1 activity is inhibited by disruption of these domains in native membranes [120], suggesting that costameric lipid rafts up-regulate NKCC1 activity. Conversely, NKCC1 has been shown to interact with and inhibit the insulinregulating, L-type amino acid transporter LAT1/SLC7A5 [121], leading to decreased ERK, Akt, and mTOR signaling [113]. Costameric NKCC1 therefore could regulate ERK signaling through conformational changes or shifts in localization of other costameric proteins.
Although the full mechanism of SC-mediated signaling remains to be elucidated, the candidate Sgcg interactors identified here suggest mechanisms for intervention in many myopathologies. The role of NKCC1 in promoting chloride uptake [67] may be mechanistically important because the resulting increases in cytosolic $\mathrm{Cl}^{-}$could support the higher concentrations of intracellular $\mathrm{Ca}^{++}$ responsible for muscle damage by serving as $\mathrm{Ca}^{++}$counterions [106]. The candidate interactors involved in estrogen-, TRIM25- and TP53-associated signaling pathways also suggest cross-talk with anti-apoptotic pathways [122] and additional avenues for further investigation, including potential relevance to NKCC1-based genetic disorders [123].

\section{Conclusions}

Our results indicate that NKCC1 associates directly or indirectly with the sarcoglycan complex in mouse muscle. Inhibition of NKCC1 transport activity is consistent with a role in sarcoglycan-mediated regulation of ERK1/2 signaling after ECC. Pathway analysis of other candidate Sgcg interactors suggests survival signaling mechanisms that may be manipulatable clinical targets in limb girdle muscular dystrophy and other myopathologies.

\begin{abstract}
Abbreviations
ATP: Adenosine triphosphate; $\mathrm{Ca}^{++}$: Calcium ions; CSK: Cytoskeletal buffer; DGC: Dystrophin glycoprotein complex; DTT: Dithiothreitol; ECC: Eccentric contractions; ECL: Enhanced chemiluminescence; ECM: Extracellular matrix; EDL: Extensor digitorum longus muscle; ERK1/2: Extracellular regulated kinases 1 and 2; GST: Glutathione s-transferase; IB: Immunoblot; IF: Immunofluorescence; IP: Immunoprecipitation; IPA: Ingenuity Pathway Analysis software; LAT1/SLC7A5: Large neutral amino acids transporter, small subunit 1; LC-MS/ MS: Liquid chromatography with tandem mass spectrometry; LGMD: Limb girdle muscular dystrophy; MAPK: Mitogen-activated protein kinases; mAV: Murine archvillin; MEK1/2: MAPK/ERK kinase 1 and 2; MOPS: 3-Morpholinopropane-1-sulfonic acid; MYPT2/PPP1r12b: Myosin phosphatase target subunit 2/protein phosphatase 1, regulatory subunit $12 B$; NKCC1: Sodium potassium chloride co-transporter 1; PLA: Proximity ligation assay; PMSF: Phenylmethylsulfonyl fluoride; PP1 $\beta$ : Serine/threonine protein phosphatase catalytic subunit 1 beta; (P/T)-ERK1/2: (phospho/total) ERK1/2; RH30: SJC-RH30 human rhabdomyosarcoma cells; RIPA: Radioimmunoprecipitation assay buffer; Rx: Relaxation buffer; SLC12A2: Solute carrier family 12 member 2; SC: Sarcoglycan complex; Sgca: Alpha-sarcoglycan; Sgcb: Beta-sarcoglycan; Sgcd: Delta-sarcoglycan; Sgcg: Gamma-sarcoglycan; Svil, SV: Supervillin; TA: Tibialis anterior muscle; WCL: Whole cell lysate.
\end{abstract}

\section{Supplementary Information}

The online version contains supplementary material available at https://doi. org/10.1186/s13395-021-00285-2.

Additional file 1: Table S1. Primers used in this study.

Additional file 2: Figure S1. Validation of new and commercial polyclonal antibodies and expression of SC proteins in RH30 cells. New affinitypurified rabbit (Rb) antibodies were generated against murine archvillin (mAV) residues 121-568 (anti-mAV) and against Sgcg residues 72-290 (anti-Sgcg). Full-length immunoblots show that (A) anti-mAV and (B) our anti-Sgcg are specific for the expected $\sim 245-\mathrm{kDa}$ and $\sim 35-\mathrm{kDa}$ bands in 
mouse muscle, but extra bands are present in RH30 cell lysates. Specificity of the major bands is confirmed by their absence from muscles lacking the immunogen and by increased staining in transfected cells. (C) Staining is similar for Proteintech Group (PTG) anti-Sgcg antibody. Immunoblots of gastrocnemius muscle proteins from wild-type (WT, lanes 1) and Svit- or $\mathrm{Sgcg}^{-/}$muscles (lanes 2), untransfected RH30 cells (lanes 3) and RH30 cells transfected with vectors encoding Sgcg- Flag (lanes 4), Sgcd- Flag (lanes 5) or Sgcb- Flag (lanes 6). Exogenous expression in RH30 cells was confirmed for Flag-tagged (B, C) Sgcg, (D) Sgcb and (E) Sgcd. No endogenous sarcoglycans were detected in RH30 cells. (B, C) The same blots used for anti-Sgcg staining were stripped and re-probed with anti-actin as a loading control. No cross-reactivity of anti-Sgcg was observed with the structurally similar Sgcd protein. Minor immunoreactive higher molecular mass bands at $\sim 70 \mathrm{kDa}$ and $\sim 160 \mathrm{kDa}$ in RH30 cells are variably present. These larger bands may represent SDS-resistant complexes of sarcoglycans $[49,63]$. Positions of molecular mass markers in $\mathrm{kDa}$ are shown on the left. Arrows denote specific bands of the expected size.

Additional file 3: Table S2. Samples report for all proteins identified in immunoprecipitates from Rx buffer. Normalized weighted spectral counts from all immunoprecipitations performed in Rx buffer; dataset before manual curating, as described in Methods.

Additional file 4: Table S3. Samples report for all proteins identified in immunoprecipitates from RIPA1 buffer. Normalized weighted spectral counts from all immunoprecipitations performed in RIPA1 buffer; dataset before manual curating, as described in Methods.

Additional file 5: Figure S2. Phase contrast micrographs of gastrocnemius muscle membrane fractions. Fractions, referenced as described in Fig. 1A, from (A, D, G, J) C57BL/6 (WT), (B, E, H, K) Sgcg ${ }^{-/}$and (C, F, I, L) Svil/mice were visualized by phase-contrast microscopy. Large membrane fragments (arrows) were observed in (A-C) total muscle extracts and (D-F) crude membrane pellets (Lane 4), but only small particulates were present in the supernatants after extraction with (G-I) Rx or (J-L) RIPA1 buffers. Bar, $0.2 \mathrm{~mm}$.

Additional file 6: Figure S3. Fractionation of selected candidate Sgcg interactors from the different muscle genotypes. Fractions from $\sim 100 \mathrm{mg}$ skeletal muscle were extracted as shown in Fig. 1, immunoblotted and probed for the Sgcg candidate interactors PP1 $\beta$ (Life Span Biosciences), MYPT2 (Proteintech Group), or NKCC1 (Alomone Laboratories). All fractions are normalized as in Fig. 2. Each immunoblot is representative of 3 (Sgcg-/-, Svil-/-) or 4 (WT) independent biological replicates. The multiple bands for NKCC1 are a consequence of differential glycosylation [74, 75].

Additional file 7: Figure S4. Validation of rabbit (Rb) polyclonal antibodies against Sgcg for immunofluorescence microscopy. Evaluation of Proteintech Group Inc. (PTG, A-D) and our new affinity-purified (ours, E-H) anti-Sgcg antibodies for immunofluorescence microscopy, as compared to nonspecific Rb lgG (I-L), in RH30 cells transfected with plasmids encoding Sgcg-Flag. Both anti-Sgcg antibodies specifically recognize structures stained with anti-Flag (arrows). Overlapping red and green signals appear as yellow.

Additional file 8: Figure S5. Validation of rabbit (Rb) polyclonal antibodies against NKCC1. (A, C) Full-length immunoblots of mouse muscle and RH30 cells with and without transfected HA-CFP-NKCC1; (B, D) Anti-NKCC1 (panels a, d; red in merges) and murine ( $M$ ) anti-HA staining (panels b, e; blue in merges) in RH30 cells transfected with HA-CFP-NKCC1. AntiNKCC1 antibodies were from (A, B) Alomone Labs, \#ANT-071 and (C, D) Proteintech Group, \#13884-1-AP. Both anti-NKCC1 antibodies recognized the expected bands in the $97-220 \mathrm{kDa}$ molecular mass range (A, C). Similar staining patterns have been reported previously, with the multiplicity of bands attributed to differential splicing and variations in glycosylation $[74,75]$. In immunofluorescence $(B, D)$, only the Proteintech antibody ( $\mathrm{Da}$, Dd) exhibited enhanced staining in $\mathrm{RH} 30$ cells expressing high levels of exogenous HA-CFP-NKCC1 (B, D, arrows). Overlapping signals appear as magenta in Merges (c and f). Bars, $20 \mu \mathrm{m}$.

Additional file 9: Figure S6. IF signal of AbCam rabbit monoclonal anti- PP1 $\beta$ correlates with EGFP signal from EGFP-PP1 $\beta$ or PP1 $\beta$-EGFP. (A) Full-length immunoblots of mouse muscle and $\mathrm{RH} 30$ cells with and without transfected PP1 $\beta$-EGFP, as indicated; lanes 1 and 5 show molecular mass markers. (B) Anti-PP1 $\beta$ (panels a, e; red in merges) and EGFP fluorescence (panels b, f; green in merges) in RH30 cells transfected with PP1 $\beta$ cDNA, as indicated. (A) The expected bands at $\sim 35 \mathrm{kDa}$ and $\sim 68 \mathrm{kDa}$ were observed in $\mathrm{RH} 30$ cells with only endogenous PP1 $\beta$ (lane 3 ) and after transfection with PP1 $\beta$-EGFP (lane 4). (B) Enhanced anti-PP1 $\beta$ staining was observed in $\mathrm{RH} 30$ cells expressing high levels of PP1 $\beta$ ( $B$, arrows) after fixation with either cytoskeleton buffer + paraformaldehyde (CSK-PFA) or $-20^{\circ} \mathrm{C}$ methanol (MeOH). Overlapping signals appear as yellow in Merges (d, h). Bars, $20 \mu \mathrm{m}$.

Additional file 10: Figure S7. Little or no signal overlap for exogenously expressed Sgcg and tensin 2 in RH30 cells. (A) Flag-tagged Sgcg, Sgcb and Sgcd were co-expressed with GFP-tagged human tensin 2. (B) Untagged Sgcg was co-expressed with Flag-tagged murine TenC1 / tensin 2. In both cases, Sgcg was stained with the Proteintech anti-Sgcg (Aa, Ae, Ba, $\mathrm{Bd}$, red in merges); tensin 2 was visualized using either (A) the GFP signal $(b, f$, green (g) in merges) or (B) anti-Flag antibody ( $b$, e, green in merges). (A) Actin filaments were stained with fluorescent phalloidin ( $c, g$, blue in merges). Cells with varied expression levels are shown. Sgcg signal is associated with internal structures, as well as in peripheral punctae (arrow). Note the absence of staining in the untransfected cell in panel Aa under the arrow that points to Sgcg punctae in the transfected cell above. Both human and murine tensin 2 isoforms localize in focal adhesions at the ends of actin filaments (arrowheads), but the tensin 2-enriched spots do not co-localize with the Sgcg punctae. Bars, $20 \mu \mathrm{m}$.

Additional file 11: Figure S8. Limited signal overlap for Sgcg and MYPT2 in RH30 cells. Untagged Sgcg visualized with Proteintech anti-Sgcg (a, d, g, red in merges) was transfected with Flag-MYPT2 (b, e, h, green in merges) in RH30 cells. An occasional cell showed some overlap of Flag-MYPT2 with Sgcg at internal structures (double arrows). Most Sgcg signal was associated with internal structures and peripheral punctae (arrows) while most MYPT2 localized with phalloidin-stained actin filaments (arrowheads; phalloidin staining not shown). Signal overlaps appear as yellow-orange. Bar, $20 \mu \mathrm{m}$.

Additional file 12: Figure S9. Limited signal overlap for Sgcg and EGFPtagged PP1 $\beta$ in RH30 cells. (A) Cells were co-transfected with Flag-tagged Sgcg, Sgcd, Sgcb, and the EGFP constructs shown. Sgcg was visualized with Proteintech anti-Sgcg ( $a$, e, i; red in merges); PP1 $\beta$-EGFP (b), EGFPPP1 $\beta(f)$ and EGFP $(j)$ are shown in green in merges $(d, h, l)$. Filamentous actin was visualized with AlexaFluor350 phalloidin to show cell outlines (c,g,k; blue in merges). (B) RH30 cells transfected with Flag-tagged Sgcg, Sgcd, and Sgcb were fixed (a-d) $10 \mathrm{~min}$ and (e-f) $15 \mathrm{~min}$ after a switch to hypo-osmotic media and stained with anti-Flag antibodies ( $a$, e; red in merges) and with the Abcam antibody against endogenous PP1 $\beta$ ( $b, f_{;}$ green in merges). PP1 $\beta$-associated signals were not excluded from Sgcgassociated structures, but convincing co-localizations were not observed. Nuclear DNA was visualized with DAPI (c, g; blue in merges). Bars, $20 \mu \mathrm{m}$.

Additional file 13: Figure S10. Changes in Total NKCC1 and phosphorylated (P)-NKCC1 in response to eccentric contraction (ECC) strain in WT and $\mathrm{Sgcg}^{-1 /} \mathrm{EDL}$ muscles. (A) Representative immunoblots. (B) The relative amount of glycosylated ( $170 \mathrm{kDa}$ ) NKCC1 tended to be lower after ECC of Sgcg $\%$ muscles $(P=0.06)$, but amounts of the nonglycosylated ( $130 \mathrm{kDa})$ NKCC1 polypeptide are unchanged $(P=0.87$ ). (C) Ratios of $P / T-N K C C 1$ after ECC relative to P/T-NKCC1 at rest for WT and $\mathrm{Sgcg}^{-\%}$ muscles. N $=5-6$ muscle pairs per strain. $P=0.07$. Central bars, means; error bars, S.D. Student's unpaired t-tests.

Additional file 14: Figure S11. Enlargement of the IPA diagram shown in Fig. 10

\section{Acknowledgements}

The authors gratefully acknowledge Drs. Michelle Dubuke and Xuni Li of the UMASS Mass Spectrometry Facility for sample analyses and assembly of Scaffold files. We thank the UMASS Pediatrics Confocal Core for access to the Leica SP5 (II) AOBS confocal microscope. We also thank the members of the UMASS Media Facility for expert reagent preparation and Donna Castellanos and Jennifer Capalbo for expert glassware washing. We are grateful to Dr. Elizabeth McNally for sarcoglycan plasmids.

\section{Authors' contributions}

Conception or design: EJL, ERB, TCS. Acquisition, analysis, or interpretation of data: TCS, GV, JMP, CHC, EJL, ERB, SAS. Drafted work or substantially revised it: EJL, TCS, ERB, GV, JMP. The author(s) read and approved the final manuscript. 


\section{Funding}

Supported by NIH R01-AR069660 award to ERB and EJL. The content of this report is solely the responsibility of the authors and does not necessarily represent the official views of the National Institutes of Health. The NIH played no direct role in the design, implementation, interpretation or writing of this manuscript.

\section{Availability of data and materials}

Antibody characterizations, negative data and sample reports in MS Excel format from the proteomics experiments are provided as supplementary data. The mass spectrometry proteomics data have been deposited to the ProteomeXchange Consortium via the PRIDE [124] partner repository with the dataset identifier PXD028584 and 10.6019/PXD028584. Scaffold 4.4.4 proteomics files that are the basis for Fig. 3 are available from the corresponding author, as are flash-frozen muscles from the new Svil-mutant mouse strain. Aliquots of the homemade affinity-purified anti-Sgcg and anti-mAV rabbit polyclonal antibodies also are available upon reasonable request while supplies last.

\section{Declarations}

\section{Ethics approval and consent to participate}

No human subjects. All animals used in this study were bred, housed and treated in accordance with standards set by the Animal Care and Use Committees at the University of Florida and the University of Massachusetts Medical School; protocol numbers 201909220 and A-1061, respectively. All anesthesia and euthanasia techniques conformed with the American Veterinary Medical Association Guidelines for the Euthanasia of Animals (2020).

\section{Consent for publication}

Not applicable.

\section{Competing interests}

The authors declare that they have no competing interests.

\section{Author details}

${ }^{1}$ Department of Radiology, Division of Cell Biology \& Imaging, University of Massachusetts Medical School, Worcester, MA, USA. ${ }^{2}$ Applied Physiology \& Kinesiology, College of Health \& Human Performance, University of Florida, Gainesville, FL, USA. ${ }^{3}$ Biochemistry and Molecular Pharmacology, University of Massachusetts Medical School, Worcester, MA, USA. ${ }^{4}$ Mass Spectrometry Facility, University of Massachusetts Medical School, Shrewsbury, MA, USA.

Received: 22 July 2021 Accepted: 15 December 2021

Published online: 22 January 2022

\section{References}

1. Ervasti JM, Campbell KP. A role for the dystrophin-glycoprotein complex as a transmembrane linker between laminin and actin. J Cell Biol. 1993;122(4):809-23

2. Henderson CA, Gomez CG, Novak SM, Mi-Mi L, Gregorio CC. Overview of the muscle cytoskeleton. Compr Physiol. 2017;7(3):891-944.

3. Kuppuswamy D. Importance of integrin signaling in myocyte growth and survival. Circ Res. 2002;90(12):1240-2.

4. Vachon $\mathrm{PH}, \mathrm{Xu}$ H, Liu L, Loechel F, Hayashi Y, Arahata K, et al. Integrins (alpha7beta1) in muscle function and survival. disrupted expression in merosin-deficient congenital muscular dystrophy. J Clin Invest. 1997;100(7):1870-81.

5. Barton ER, Pacak CA, Stoppel WL, Kang PB. The ties that bind: functional clusters in limb-girdle muscular dystrophy. Skelet Muscle. 2020;10(1):22.

6. Ervasti JM, Sonnemann KJ. Biology of the striated muscle dystrophinglycoprotein complex. Int Rev Cytol. 2008;265:191-225.

7. Tarakci H, Berger J. The sarcoglycan complex in skeletal muscle. Front Biosci (Landmark Ed). 2016;21:744-56.

8. Pardo JV, Siliciano JD, Craig SW. A vinculin-containing cortical lattice in skeletal muscle: transverse lattice elements ("costameres") mark sites of attachment between myofibrils and sarcolemma. Proc Natl Acad Sci USA. 1983:80(4):1008-12.
9. Bloch RJ, Gonzalez-Serratos H. Lateral force transmission across costameres in skeletal muscle. Exerc Sport Sci Rev. 2003;31(2):73-8.

10. Ervasti JM. Costameres: the Achilles' heel of herculean muscle. J Biol Chem. 2003;278(16):13591-4.

11. Peter AK, Cheng H, Ross RS, Knowlton KU, Chen J. The costamere bridges sarcomeres to the sarcolemma in striated muscle. Prog Pediatr Cardiol. 2011;31(2):83-8.

12. Sit B, Gutmann D, Iskratsch T. Costameres, dense plaques and podosomes: the cell matrix adhesions in cardiovascular mechanosensing. J Muscle Res Cell Motil. 2019;40(2):197-209.

13. Vassilopoulos S. Unconventional roles for membrane traffic proteins in response to muscle membrane stress. Curr Opin Cell Biol. 2020;65:42-9.

14. McNally EM, Pytel P. Muscle diseases: the muscular dystrophies. Annu Rev Pathol. 2007;2:87-109.

15. Nigro V, Piluso G. Spectrum of muscular dystrophies associated with sarcolemmal-protein genetic defects. Biochim Biophys Acta. 2015;1852(4):585-93

16. Hack AA, Ly CT, Jiang F, Clendenin CJ, Sigrist KS, Wollmann RL, et al. Gamma-sarcoglycan deficiency leads to muscle membrane defects and apoptosis independent of dystrophin. J Cell Biol. 1998;142(5):1279-87.

17. Taghizadeh E, Rezaee M, Barreto GE, Sahebkar A. Prevalence, pathological mechanisms, and genetic basis of limb-girdle muscular dystrophies: a review. J Cell Physiol. 2019;234(6):7874-84.

18. Barton ER. Impact of sarcoglycan complex on mechanical signal transduction in murine skeletal muscle. Am J Physiol Cell Physiol. 2006;290(2):C411-9.

19. Hack AA, Cordier L, Shoturma DI, Lam MY, Sweeney HL, McNally EM Muscle degeneration without mechanical injury in sarcoglycan deficiency. Proc Natl Acad Sci U S A. 1999;96(19):10723-8.

20. Moorwood C, Philippou A,Spinazzola J,Keyser B, MacarakEJ, BartonER. Absence of gamma-sarcoglycan alters the response of P70S6 kinase to mechanical perturbation in murine skeletal muscle. Skelet Muscle. 2014;4:13.

21. Thompson TG, Chan YM, Hack AA, Brosius M, Rajala M, Lidov HG, et al. Filamin 2 (FLN2): a muscle-specific sarcoglycan interacting protein. J Cell Biol. 2000;148(1):115-26.

22. Griffin MA, Feng $H$, Tewari $M$, Acosta $P$, Kawana $M$, Sweeney $H L$, et al. Gamma-sarcoglycan deficiency increases cell contractility, apoptosis and mapk pathway activation but does not affect adhesion. J Cell Sci. 2005;118(Pt 7):1405-16.

23. Burr AR, Millay DP, Goonasekera SA, Park KH, Sargent MA, Collins J, et al. $\mathrm{Na}+$ dysregulation coupled with $\mathrm{Ca} 2+$ entry through NCX1 promotes muscular dystrophy in mice. Mol Cell Biol. 2014;34(11):1991-2002.

24. Barton ER. Restoration of gamma-sarcoglycan localization and mechanical signal transduction are independent in murine skeletal muscle. J Biol Chem. 2010;285(22):17263-70.

25. Blandin G, Marchand S, Charton K, Daniele N, Gicquel E, Boucheteil JB, et al. A human skeletal muscle interactome centered on proteins involved in muscular dystrophies: LGMD interactome. Skelet Muscle. 2013;3(1):3.

26. Spinazzola JM, Smith TC, Liu M, Luna EJ, Barton ER. Gamma-sarcoglycan is required for the response of archvillin to mechanical stimulation in skeletal muscle. Hum Mol Genet. 2015;24(9):2470-81.

27. Oh SW, Pope RK, Smith KP, Crowley JL, NebI T, Lawrence JB, et al. Archvillin, A muscle-specific isoform of supervillin, is an early expressed component of the costameric membrane skeleton. J Cell Sci. 2003;116(Pt. 11):2261-75.

28. Hedberg-Oldfors C, Meyer R, Nolte K, Abdul Rahim Y, Lindberg C, Karason K, et al. Loss of supervillin causes myopathy with myofibrillar disorganization and autophagic vacuoles. Brain. 2020;143(8):2406-20.

29. Boyer JG, Prasad V, Song T, Lee D, Fu X, Grimes KM, et al. ERK1/2 signaling induces skeletal muscle slow fiber-type switching and reduces muscular dystrophy disease severity. Jci. Insight. 2019;5.

30. Gangopadhyay SS, Kengni E, Appel S, Gallant C, Kim HR, Leavis P, et al. Smooth muscle archvillin is an ERK scaffolding protein. J Biol Chem. 2009:284(26):17607-15.

31. Pestonjamasp KN, Pope RK, Wulfkuhle JD, Luna EJ. Supervillin (P205): a novel membrane-associated, F-actin-binding protein in the villin/gelsolin superfamily. J Cell Biol. 1997;139(5):1255-69.

32. Fang $Z Y$, Luna EJ. Supervillin-mediated suppression of $P 53$ protein enhances cell survival. J Biol Chem. 2013;288(11):7918-29. 
33. Takizawa N, Smith TC, NebI T, Crowley JL, Palmieri SJ, Lifshitz LM, et al. Supervillin modulation of focal adhesions involving TRIP6/ZRP-1. J Cell Biol. 2006;174(3):447-58.

34. Takizawa N, Ikebe R, Ikebe M, Luna EJ. Supervillin slows cell spreading by facilitating myosin II activation at the cell periphery. J Cell Sci. 2007;120(Pt 21):3792-803

35. Nebl T, Pestonjamasp KN, Leszyk JD, Crowley JL, Oh SW, Luna EJ. Proteomic analysis of a detergent-resistant membrane skeleton from neutrophil plasma membranes. J Biol Chem. 2002;277(45):43399-409.

36. Gao QQ, Wyatt E, Goldstein JA, LoPresti P, Castillo LM, Gazda A, et al. Reengineering a transmembrane protein to treat muscular dystrophy using exon skipping. J Clin Invest. 2015;125(11):4186-95.

37. Laemmli UK. Cleavage of structural proteins during the assembly of the head of bacteriophage T4. Nature (Lond). 1970;227:680-5.

38. Bornhorst JA, Falke JJ. Purification of proteins using polyhistidine affinity tags. Methods Enzymol. 2000;326:245-54.

39. Nguyen Thi M, Cartwright AJ, Morris GE, Love DR, Bloomfield JF, Davies KE. Monoclonal antibodies against defined regions of the muscular dystrophy protein, dystrophin. Febs Lett. 1990;262(2):237-40.

40. Hughes SM, Cho M, Karsch-Mizrachi I, Travis M, Silberstein L, Leinwand LA, et al. Three slow myosin heavy chains sequentially expressed in developing mammalian skeletal muscle. Dev Biol. 1993;158(1):183-99.

41. Webster C, Silberstein L, Hays AP, Blau HM. Fast muscle fibers are preferentially affected in duchenne muscular dystrophy. Cell. 1988;52(4):503-13.

42. Cong L, Ran FA, Cox D, Lin S, Barretto R, Habib N, et al. Multiplex genome engineering using Crispr/Cas systems. Science. 2013;339(6121):819-23.

43. Yamamoto N,YamashitaY,YoshiokaY,NishiumiS,Ashida H. Rapid preparation of a plasma membrane fraction: western blot detection of translocated glucose transporter 4 from plasma membrane of muscle and adipose cells and tissues. Curr Protoc Protein Sci. 2016;85:29.18.1-29.18.12.

44. White DC. Rigor contraction and the effect of various phosphate compounds on glycerinated insect flight and vertebrate muscle. J Physiol. 1970:208(3):583-605.

45. Del Buono BJ, Luscinskas FW, Simons ER. Preparation and characterization of plasma membrane vesicles from human polymorphonuclear leukocytes. J Cell Physiol. 1989;141:636-44.

46. Ohlendieck K, Ervasti JM, Snook JB, Campbell KP. Dystrophin-glycoprotein complex is highly enriched in isolated skeletal muscle sarcolemma. J Cell Biol. 1991;112(1):135-48.

47. Song KS, Li S, Okamoto T, Quilliam LA, Sargiacomo M, Lisanti MP. Co-purification and direct interaction of ras with caveolin, an integral membrane protein of caveolae microdomains. Detergent-free purification of caveolae microdomains. J Biol Chem. 1996;271(16):9690-7.

48. Joe MK, Kee C, Tomarev SI. Myocilin interacts with syntrophins and is member of dystrophin-associated protein complex. J Biol Chem. 2012;287(16):13216-27.

49. Arias-Calderon M, Almarza G, Diaz-Vegas A, Contreras-Ferrat A, Valladares D, Casas M, et al. Characterization of a multiprotein complex involved in excitation-transcription coupling of skeletal muscle. Skelet Muscle. 2016;6:15.

50. Nigro V, Savarese M. Genetic basis of limb-girdle muscular dystrophies: the 2014 update. Acta Myol. 2014;33(1):1-12.

51. Holt K, Campbell KP. Assembly of the sarcoglycan complex. Insights for muscular dystrophy. J Biol Chem. 1998;273(52):34667-70.

52. Fechheimer M, Daiss JL, Cebra JJ. Interaction of immunoglobulin with actin. Mol Immunol. 1979;16:881-8.

53. Lesage B, Beullens M, Nuytten M, Van Eynde A, Keppens S, Himpens B, et al. Interactor-mediated nuclear translocation and retention of protein phosphatase-1. J Biol Chem. 2004;279(53):55978-84.

54. Trinkle-Mulcahy L, Sleeman JE, Lamond AI. Dynamic targeting of protein phosphatase 1 within the nuclei of living mammalian cells. J Cell Sci. 2001;114(Pt 23):4219-28.

55. Clark K, Howe JD, Pullar CE, Green JA, Artym W, Yamada KM, et al. Tensin 2 modulates cell contractility in 3D collagen gels through The Rhogap Dlc1. J Cell Biochem. 2010;109(4):808-17.

56. Somasekharan S, Monette MY, Forbush B. Functional expression of human NKCC1 from a synthetic cassette-based cDNA: introduction of extracellular epitope tags and removal of cysteines. PLoS One. 2013:8(12):E82060.
57. Smith TC, Fang Z, Luna EJ. Novel interactors and a role for supervillin in early cytokinesis. Cytoskeleton (Hoboken). 2010;67(6):346-64.

58. Spinazzola JM. Gamma-sarcoglycan mediated mechanotransduction in skeletal muscle [Ph.D.]. Philadelphia: University Of Pennsylvania; 2015.

59. Smith TC, Saul RG, Barton ER, Luna EJ. Generation and characterization of monoclonal antibodies that recognize human and murine supervillin protein isoforms. PLoS One. 2018;13(10):E0205910.

60. Wagner S, Chiosea S, Nickerson JA. The spatial targeting and nuclear matrix binding domains of SRm160. Proc Natl Acad Sci U S A. 2003;100(6):3269-74.

61. Pollard TD. A guide to simple and informative binding assays. Mol Biol Cell. 2010;21(23):4061-7.

62. Moorwood C, Liu M, Tian Z, Barton ER. Isometric and eccentric force generation assessment of skeletal muscles isolated from murine models of muscular dystrophies. J Vis Exp. 2013;(71):E50036.

63. Draviam RA, Wang B, Shand SH, Xiao X, Watkins SC. Alpha-sarcoglycan is recycled from the plasma membrane in the absence of sarcoglycan complex assembly. Traffic. 2006;7(7):793-810.

64. Shi W, Chen Z, Schottenfeld J, Stahl RC, Kunkel LM, Chan YM. Specific assembly pathway of sarcoglycans is dependent on beta- and deltasarcoglycan. Muscle Nerve. 2004;29(3):409-19.

65. Yoshida M, Suzuki A, Yamamoto H, Noguchi S, Mizuno Y, Ozawa E. Dissociation of the complex of dystrophin and its associated proteins into several unique groups by N-octyl beta-D-glucoside. Eur J Biochem. 1994;222(3):1055-61.

66. Gagnon KB, Delpire E. Multiple pathways for protein phosphatase 1 (PP1) Regulation of Na-K-2Cl cotransporter (NkCC 1) function: the $\mathrm{N}$-terminal tail of the $\mathrm{Na}-\mathrm{K}-2 \mathrm{Cl}$ cotransporter serves as a regulatory scaffold for Ste20-related proline/alanine-rich kinase (SPAK) and Pp1. J Biol Chem. 2010;285(19):14115-21.

67. Delpire $\mathrm{E}$, Gagnon $\mathrm{KB} . \mathrm{Na}^{+}-\mathrm{K}^{+}-2 \mathrm{Cl}^{-}$cotransporter (NKCC) physiological function in nonpolarized cells and transporting epithelia. Compr Physiol. 2018;8(2):871-901.

68. Grassie ME, Moffat LD, Walsh MP, MacDonald JA. The myosin phosphatase targeting protein (MYPT) family: a regulated mechanism for achieving substrate specificity of the catalytic subunit of protein phosphatase type 1delta. Arch Biochem Biophys. 2011;510(2):147-59.

69. Moorhead G, Johnson D, Morrice N, Cohen P.The major myosin phosphatase in skeletal muscle is a complex between the beta-isoform of protein phosphatase 1 and the MYPT2 gene product. Febs Lett. 1998;438(3):141-4.

70. Okamoto R, Kato T, Mizoguchi A, Takahashi N, Nakakuki T, Mizutani $\mathrm{H}$, et al. Characterization and function of MYPT2, a target subunit of myosin phosphatase in heart. Cell Signal. 2006:18(9):1408-16.

71. Blangy A. Tensins are versatile regulators of rho GTPase signalling and cell adhesion. Biol Cell. 2017:109(3):115-26.

72. Eto M, Kirkbride J, Elliott E, Lo SH, Brautigan DL. Association of the tensin N-terminal protein-tyrosine phosphatase domain with the alpha isoform of protein phosphatase-1 in focal adhesions. J Biol Chem. 2007;282(24):17806-15.

73. Georgiadou M, Ivaska J. Tensins: Bridging AMP-activated protein kinase with integrin activation. Trends Cell Biol. 2017;27(10):703-11.

74. Gehmlich K, Pinotsis N, Hayess K, van der Ven PF, Milting H, El Banayosy A, et al. Paxillin and ponsin interact in nascent costameres of muscle cells. J Mol Biol. 2007:369(3):665-82.

75. Otey CA, Rachlin A, Moza M, Arneman D, Carpen O. The palladin/ myotilin/myopalladin family of actin-associated scaffolds. Int Rev Cytol. 2005;246:31-58

76. Nezu A, Parvin MN, Turner RJ. A conserved hydrophobic tetrad near the $\mathrm{C}$ terminus of the secretory $\mathrm{Na}+-\mathrm{K}+-2 \mathrm{Cl}$ - cotransporter (NKCC1) is required for its correct intracellular processing. J Biol Chem. 2009:284(11):6869-76

77. Singh R, Almutairi MM, Pacheco-Andrade R, Almiahuob MY, Di Fulvio M. Impact of hybrid and complex $\mathrm{N}$-glycans on cell surface targeting of the endogenous chloride cotransporter SLC12A2. Int J Cell Biol. 2015:2015:505294.

78. Zhang M, Truscott J, Davie J. Loss of MEF2D expression inhibits differentiation and contributes to oncogenesis in rhabdomyosarcoma cells. Mol Cancer. 2013;12(1):150.

79. Bannert N, Vollhardt K, Asomuddinov B, Haag M, Konig H, Norley S, et al. PDZ domain-mediated interaction of interleukin-16 precursor proteins with myosin phosphatase targeting subunits. J Biol Chem. 2003:278(43):42190-9. 
80. McMahon KA, Wu Y, Gambin Y, Sierecki E, Tillu VA, Hall T, et al. Identification of intracellular cavin target proteins reveals cavin-PP1alpha interactions regulate apoptosis. Nat Commun. 2019;10(1):3279.

81. Wong JA, Fu L, Schneider EG, Thomason DB. Molecular and functional evidence for $\mathrm{Na}(+)-\mathrm{K}(+)-2 \mathrm{Cl}(-)$ cotransporter expression in rat skeletal muscle. Am J Physiol. 1999;277(1):R154-61.

82. Hong T, Shaw RM. Cardiac T-tubule microanatomy and function. Physiol Rev. 2017;97(1):227-52.

83. Murphy RM, Mollica JP, Lamb GD. Plasma membrane removal in rat skeletal muscle fibers reveals caveolin-3 hot-spots at the necks of transverse tubules. Exp Cell Res. 2009;315(6):1015-28.

84. Mandai S, Furukawa S, Kodaka M, Hata Y, Mori T, Nomura N, et al. Loop diuretics affect skeletal myoblast differentiation and exerciseinduced muscle hypertrophy. Sci Rep. 2017;7:46369.

85. Monette MY, Forbush B. Regulatory activation is accompanied by movement in the $\mathrm{C}$ terminus of the $\mathrm{Na}-\mathrm{K}-\mathrm{Cl}$ cotransporter (NKCC1). J Biol Chem. 2012;287(3):2210-20.

86. Vitari AC, Thastrup J, Rafiqi F, Deak M, Morrice NA, Karlsson HK, et al. Functional interactions of the SPAK/OSR1 kinases with their upstream activator wnk1 and downstream substrate NKCC1. Biochem J. 2006;397(1):223-31.

87. Müller T, Schrotter A, Loosse C, Helling S, Stephan C, Ahrens M, et al. Sense and nonsense of pathway analysis software in proteomics. J Proteome Res. 2011;10(12):5398-408.

88. Spence HJ, Dhillon AS, James M, Winder SJ. Dystroglycan, a scaffold for the ERK-MAP kinase cascade. Embo Rep. 2004;5(5):484-9.

89. Allard B. From excitation to intracellular $\mathrm{Ca}(2+)$ movements in skeletal muscle: basic aspects and related clinical disorders. Neuromuscul Disord. 2018;28(5):394-401.

90. Estojak J, Brent R, Golemis EA. Correlation of two-hybrid affinity data with in vitro measurements. Mol Cell Biol. 1995;15(10):5820-9.

91. Jameson SC, Hogquist KA, Bevan MJ. Positive selection of thymocytes. Annu Rev Immunol. 1995;13:93-126.

92. Ivashkiv LB. How ITAMs inhibit signaling. Sci Signal. 2011;4(169):Pe20.

93. Xie ZR, Chen J, Wu Y. Linking 3D and 2D binding kinetics of membrane proteins by multiscale simulations. Protein Sci. 2014;23(12):1789-99.

94. Brautigan DL, Shenolikar S. Protein serine/threonine phosphatases: keys to unlocking regulators and substrates. Annu Rev Biochem. 2018;87:921-64.

95. Imamura M, Araishi K, Noguchi S, Ozawa E. A sarcoglycan-dystroglycan complex anchors Dp116 and utrophin in the peripheral nervous system. Hum Mol Genet. 2000;9(20):3091-100.

96. Yoon J, Johnson E, Xu R, Martin LT, Martin PT, Montanaro F. Comparative proteomic profiling of dystroglycan-associated proteins in wild type, Mdx, And Galgt2 transgenic mouse skeletal muscle. J Proteome Res. 2012:11(9):4413-24.

97. Dowling P, Gargan S, Murphy S, Zweyer M, Sabir H, Swandulla D, et al The dystrophin node as integrator of cytoskeletal organization, lateral force transmission, fiber stability and cellular signaling in skeletal muscle. Proteomes. 2021;9(1):9.

98. Gosmanov AR, Lindinger MI, Thomason DB. Riding the tides: $\mathrm{K}+\mathrm{con}$ centration and volume regulation by muscle $\mathrm{Na}+-\mathrm{K}+-2 \mathrm{Cl}$ - cotransport activity. News Physiol Sci. 2003;18:196-200.

99. Shen B, Fu J, Guo J, Zhang J, Wang X, Pan X, et al. Role of $\mathrm{Na}^{+}-\mathrm{K}^{+}-2 \mathrm{Cl}^{-}$ cotransporter 1 in phenylephrine-induced rhythmic contraction in the mouse aorta: regulation of $\mathrm{Na}^{+}-\mathrm{K}^{+}-2 \mathrm{Cl}^{-}$cotransporter 1 by $\mathrm{Ca} 2+$ sparks and KCa channels. Cell Physiol Biochem. 2015;37(2):747-58

100. Wu F, Mi W, Cannon SC. Beneficial effects of bumetanide in a Cav1.1R528h mouse model of hypokalaemic periodic paralysis. Brain. 2013;136(Pt 12):3766-74.

101. Wu F, Mi W, Cannon SC. Bumetanide prevents transient decreases in muscle force in murine hypokalemic periodic paralysis. Neurology. 2013;80(12):1110-6.

102. Schobel N, Radtke D, Lubbert M, Gisselmann G, Lehmann R, Cichy A, et al. Trigeminal ganglion neurons of mice show intracellular chloride accumulation and chloride-dependent amplification of capsaicininduced responses. PLos One. 2012;7(11):E48005.

103. Witte M, Reinert T, Dietz B, Nerlich J, Rubsamen R, Milenkovic I. Depolarizing chloride gradient in developing cochlear nucleus neu rons: underlying mechanism and implication for calcium signaling. Neuroscience. 2014:261:207-22.
104. Tabuchi A, Eshima H, Tanaka Y, Nogami S, Inoue N, Sudo M, et al. Regional differences in $\mathrm{Ca}(2+)$ entry along the proximal-middle-distal muscle axis during eccentric contractions in rat skeletal muscle. J Appl Physiol (1985). 2019;127(3):828-37.

105. Allen DG, Gervasio OL, Yeung EW, Whitehead NP. Calcium and the damage pathways in muscular dystrophy. Can J Physiol Pharmacol. 2010;88(2):83-91.

106. Burr AR, Molkentin JD. Genetic evidence in the mouse solidifies the calcium hypothesis of myofiber death in muscular dystrophy. Cell Death Differ. 2015;22(9):1402-12.

107. Bueno OF, De Windt LJ, Tymitz KM, Witt SA, Kimball TR, Klevitsky $R$, et al. The MEK1-ERK1/2 signaling pathway promotes compensated cardiac hypertrophy in transgenic mice. Embo J. 2000;19(23):6341-50.

108. Kehat I, Molkentin JD. Extracellular signal-regulated kinase 1/2 (ERK1/2) signaling in cardiac hypertrophy. Ann N Y Acad Sci. 2010;1188:96-102.

109. Liedtke CM, Cole TS. Activation of NKCC1 by hyperosmotic stress in human tracheal epithelial cells involves PKC-delta and ERK. Biochim Biophys Acta. 2002;1589(1):77-88.

110. Panet $\mathrm{R}$, Eliash $\mathrm{M}$, Atlan $\mathrm{H}$. Na+/K+/Cl- cotransporter activates MAPkinase cascade downstream to protein kinase $C$, and upstream to MEK. J Cell Physiol. 2006;206(3):578-85.

111. Panet $\mathrm{R}$, Eliash $\mathrm{M}$, Pick M, Atlan $\mathrm{H}$. Na(+)/K(+)/Cl(-) cotransporter activates mitogen-activated protein kinase in fibroblasts and lymphocytes. J Cell Physiol. 2002;190(2):227-37.

112. Cai L, Du T, Song D, Li B, Hertz L, Peng L. Astrocyte ERK phosphorylation precedes $\mathrm{K}(+)$-induced swelling but follows hypotonicityinduced swelling. Neuropathology. 2011;31(3):250-64.

113. Demian WL, Persaud A, Jiang C, Coyaud E, Liu S, Kapus A, et al. The ion transporter NKCC 1 links cell volume to cell mass regulation by suppressing mTORC1. Cell Rep. 2019;27(6):1886-96.E6.

114. Lu KT, Cheng NC, Wu CY, Yang YL. NKCC 1-mediated traumatic brain injury-induced brain edema and neuron death via Raf/MEK/MAPK Cascade. Crit Care Med. 2008;36(3):917-22.

115. Wang Z, Bildin VN, Yang H, Capo-Aponte JE, Yang Y, Reinach PS. Dependence of corneal epithelial cell proliferation on modulation of interactions between ERK1/2 and NKCC1. Cell Physiol Biochem. 2011;28(4):703-14.

116. Cong D, Zhu W, Kuo JS, Hu S, Sun D. Ion transporters in brain tumors. Curr Med Chem. 2015;22(10):1171-81.

117. Woodman SE, Park DS, Cohen AW, Cheung MW, Chandra M, Shirani J, et al. Caveolin-3 knock-out mice develop a progressive cardiomyopathy and show hyperactivation of the P42/44 MAPK cascade. J Biol Chem. 2002;277(41):38988-97.

118. Baenziger JE, Domville JA, Therien JPD. The role of cholesterol in the activation of nicotinic acetylcholine receptors. Curr Top Membr. 2017;80:95-137.

119. Morales-Lazaro SL, Rosenbaum T. Multiple mechanisms of regulation of transient receptor potential ion channels by cholesterol. Curr Top Membr. 2017:80:139-61.

120. Hartmann AM, Blaesse P, Kranz T, Wenz M, Schindler J, Kaila K, et al. Opposite effect of membrane raft perturbation on transport activity of KCC2 And NKCC1. J Neurochem. 2009;111(2):321-31.

121. Javed K, Fairweather $\mathrm{SJ}$. Amino acid transporters in the regulation of insulin secretion and signalling. Biochem Soc Trans. 2019;47(2):571-90.

122. Boland R, Vasconsuelo A, Milanesi L, Ronda AC, de Boland AR. 17betaestradiol signaling in skeletal muscle cells and its relationship to apoptosis. Steroids. 2008;73(9-10):859-63.

123. Koumangoye R, Bastarache L, Delpire E. NKCC1: newly found as a human disease-causing ion transporter. Function. 2021;2(1):Zqaa028.

124. Vizcaino JA, Deutsch, EW, Wang R, Csordas A, Reisinger F, Ríos D, et al. ProteomeXchange provides globally coordinated proteomics data submission and dissemination. Nature Biotech. 2014;32:223-6.

\section{Publisher's Note}

Springer Nature remains neutral with regard to jurisdictional claims in published maps and institutional affiliations. 